\title{
Equilibrium and matching under price controls
}

Citation for published version (APA):

Herings, P. J-J. (2018). Equilibrium and matching under price controls. Journal of Economic Theory, 177, 222-244. https://doi.org/10.1016/j.jet.2018.06.004

Document status and date:

Published: 01/09/2018

DOI:

10.1016/j.jet.2018.06.004

Document Version:

Publisher's PDF, also known as Version of record

Document license:

Taverne

Please check the document version of this publication:

- A submitted manuscript is the version of the article upon submission and before peer-review. There can be important differences between the submitted version and the official published version of record.

People interested in the research are advised to contact the author for the final version of the publication, or visit the DOI to the publisher's website.

- The final author version and the galley proof are versions of the publication after peer review.

- The final published version features the final layout of the paper including the volume, issue and page numbers.

Link to publication

\footnotetext{
General rights rights.

- You may freely distribute the URL identifying the publication in the public portal. please follow below link for the End User Agreement:

www.umlib.nl/taverne-license

Take down policy

If you believe that this document breaches copyright please contact us at:

repository@maastrichtuniversity.nl

providing details and we will investigate your claim.
}

Copyright and moral rights for the publications made accessible in the public portal are retained by the authors and/or other copyright owners and it is a condition of accessing publications that users recognise and abide by the legal requirements associated with these

- Users may download and print one copy of any publication from the public portal for the purpose of private study or research.

- You may not further distribute the material or use it for any profit-making activity or commercial gain

If the publication is distributed under the terms of Article $25 \mathrm{fa}$ of the Dutch Copyright Act, indicated by the "Taverne" license above, 


\title{
Equilibrium and matching under price controls
}

\author{
P. Jean-Jacques Herings \\ Department of Economics, Maastricht University, P.O. Box 616, 6200 MD, Maastricht, the Netherlands \\ Received 21 October 2016; final version received 7 June 2018; accepted 11 June 2018 \\ Available online 18 June 2018
}

\begin{abstract}
The paper considers a matching with contracts model in the presence of price controls. The model contains two important streams in the matching literature, those with and those without monetary transfers, as special cases. An adjustment process that ends with a stable outcome is presented. The paper presents a notion of competitive equilibrium, called Drèze equilibrium, and shows Drèze equilibrium allocations to be equivalent to allocations induced by stable outcomes. We therefore have an equivalence that is valid with and without monetary transfers as well as when monetary transfers are limited.
\end{abstract}

(c) 2018 Elsevier Inc. All rights reserved.

JEL classification: C71; C78; D45; D51

Keywords: Price controls; Matching; Stable outcomes; Competitive equilibrium; Drèze equilibrium

\section{Introduction}

Although standard analysis assumes prices to be completely flexible, price controls and price regulations are actually very common, see, e.g., Nguyen and Whalley (1990) and Levy (1991). Typical examples include minimum wages in the labor market and upper bounds on rents in housing markets.

From a substantive point of view, the analysis of competitive models with price controls is very different from the one without price controls, caused by the fact that due to the price con-

\footnotetext{
I I would like to thank Tommy Andersson, Péter Biró, Francis Bloch, Péter Csóka, Gabrielle Demange, Jacques Drèze, Michael Greinecker, Dolf Talman, Fernando Vega-Redondo, Alex Teytelboym, Markus Walzl, and Alexander Westkamp for their very helpful comments on this paper.

E-mail address: P.Herings@ maastrichtuniversity.nl.
} 
trols markets are prevented from clearing. As a consequence, not only prices adjust to clear markets, but so do quantities in the form of supply and demand rationing. Typical examples are unemployment on the labor market and waiting lists in the social housing sector. Unlike competitive equilibria in models without price controls, which are Pareto efficient, equilibria in models with price controls are typically Pareto inefficient, even when restricting attention to allocations that are consistent with the given price controls (Herings and Konovalov, 2009).

Many of the examples of markets with price controls, like the housing market or the labor market, are characterized by extensive heterogeneity. An alternative to competitive analysis is therefore to use concepts from matching theory. This paper compares both approaches in a one-to-one matching with contracts model in the spirit of Hatfield and Milgrom (2005), where contract prices may be subject to price controls. Buyers and sellers trade commodities by signing contracts, where a contract specifies a buyer, a seller, a commodity, and a price. The price of a contract is subject to a price floor and a price ceiling, and therefore restricted to belong to some interval. The price floor is allowed to be equal to minus infinity and the price ceiling may be equal to plus infinity.

Two important streams in the matching literature, those with and those without monetary transfers, can be seen as special cases. The case without monetary transfers like the marriage problem of Gale and Shapley (1962) follows by specifying both the lower and the upper bound on the price to be equal to zero. The case with unrestricted monetary transfers like the assignment model of Shapley and Shubik (1971) follows by setting the interval of feasible prices equal to the set of real numbers. Other special cases are Talman and Yang (2008) and Andersson and Svensson (2014), who consider the assignment model with price controls, and Hatfield et al. (2012, 2016), who study how the presence of price controls leads to quality competition.

An alternative way to bridge models in the literatures with and without monetary transfers is presented in Jaffe and Kominers (2014). They consider a model where transfers are subject to taxation. The model with tax rates equal to zero corresponds to the standard model with monetary transfers and the model with tax rates equal to one to the model without monetary transfers.

There are several papers that have established a connection between stable outcomes and competitive equilibria for the case of unlimited monetary transfers. Shapley and Shubik (1971) consider the assignment game with transferable utility and prove that the set of stable payoffs coincides with the set of competitive equilibrium payoffs. Kaneko (1982) and Quinzii (1984) extend this result to the case where utility functions are not assumed to be quasi-linear. When allowing for personalized prices and assuming quasi-linear utility functions, such a result can be extended to many-to-many matching set-ups or even trading networks as has been demonstrated by Hatfield et al. (2013).

The equivalence results are invariably derived in a setting with unlimited monetary transfers, or, equivalently, absence of price controls. When discussing the connection between competitive markets and stability in the context of the marriage model, Shapley and Scarf (1974), p. 35, remark that: "It does not appear to be possible to set up a conventional market for this model, in such a way that a competitive price equilibrium will exist and lead to an allocation in the core." This paper presents a fully general equivalence between stable outcomes and competitive equilibria. This equivalence boils down to the equivalence with competitive equilibria in the absence of price controls, presents a completely new result for cases with no monetary transfers like the marriage problem, and also deals with all intermediate cases like wage or rent controls. Our concept of competitive equilibrium in the matching with contracts model under price controls is an extension of the contribution by Drèze (1975), who introduces price rigidities in the standard 
general equilibrium framework with divisible commodities. We refer to this concept as Drèze equilibrium.

We prove the existence of stable outcomes and, by the equivalence result, of Drèze equilibria by means of an adjustment process in the style of Gale and Shapley (1962), Crawford and Knoer (1981), Kelso and Crawford (1982), Demange et al. (1986), and Hatfield and Milgrom (2005). This process is shown to always terminate with a stable outcome in a discretized version of the model and we next use a limit argument. When applied to the marriage problem, the adjustment process is identical to the deferred acceptance algorithm of Gale and Shapley (1962). When applied to the assignment model, the adjustment process is identical to the generalization of the approximate auction mechanism with personalized prices as discussed in Section V of Demange et al. (1986).

The paper is organized as follows. Section 2 presents the model of matching with contracts under price controls and shows how many contributions in the literature are included as special cases. Section 3 presents and illustrates the notions of stable outcome and Drèze equilibrium. Section 4 provides an illustrating example. Section 5 shows stable outcomes to exist. Section 6 is devoted to the equivalence between allocations induced by stable outcomes and Drèze equilibrium allocations. Section 7 contains the conclusion.

\section{Matching under price controls}

There is a finite set of buyers $B$ and a finite set of sellers $S$ who trade commodities in a finite set $L$ and a good labeled 0 , which we call money. The set of all agents is $I=B \cup S$. Trade in commodities takes place by signing contracts, where a contract is an element $y=(b, s, \ell, m) \in$ $B \times S \times L \times \mathbb{R}$. A given contract $y=(b, s, \ell, m)$ involves a buyer $\beta(y)=b$, a seller $\sigma(y)=s$, a commodity $\lambda(y)=\ell$, and an amount of money $\mu(y)=m$. The set of agents involved in contract $y$ is $\iota(y)=\{\beta(y), \sigma(y)\}$. We consider a one-to-one matching set-up, so a buyer signs a contract with at most one seller and a seller with at most one buyer.

For a set of contracts $Y \subset B \times S \times L \times \mathbb{R}$, the set of contracts involving buyer $b$ is given by $Y^{b}=Y \cap \beta^{-1}(\{b\})=\{y \in Y \mid \beta(y)=b\}$ and the set of contracts involving seller $s$ by $Y^{s}=$ $Y \cap \sigma^{-1}(\{s\})=\{y \in Y \mid \sigma(y)=s\}$. Similarly, the set of contracts involving commodity $\ell$ is given by $Y_{\ell}=Y \cap \lambda^{-1}(\{\ell\})=\{y \in Y \mid \lambda(y)=\ell\}$. Throughout the paper, superscripts refer to agents and subscripts to commodities.

The amount of money involved in a contract may be subject to price controls. The price of a commodity $\ell \in L$ is subject to a price floor $\underline{p}_{\ell} \in\{-\infty\} \cup \mathbb{R}$ and a price ceiling $\bar{p}_{\ell} \in \mathbb{R} \cup\{+\infty\}$. The set of admissible prices is now equal to $P=\left\{p \in \mathbb{R}^{L} \mid p \leq p \leq \bar{p}\right\}$. To ensure that the set of admissible prices is non-empty, it is assumed that $\underline{p} \leq \bar{p}$. One extreme but highly relevant case is where no monetary transfers are allowed. This is achieved by setting $\underline{p}_{\ell}=\bar{p}_{\ell}=0$. In applications related to social housing, it is common that the rent $m_{\ell}$ to be paid for an apartment of type $\ell$ is fixed, which corresponds to setting $\underline{p}_{\ell}=\bar{p}_{\ell}=m_{\ell}$. The case with unlimited monetary transfers follows by specifying $\underline{p}_{\ell}=-\infty$ and $\overline{\bar{p}}_{\ell}=+\infty$. Both price floors and price ceilings are allowed to be negative.

In many settings, it is not possible for every buyer to supply every commodity to every seller. Some specific examples are presented later. The set of feasible trades is given by $T$, a subset of $B \times S \times L$. The set $T$ generates the set of feasible contracts $\bar{Y}$ defined as the set of all contracts with the trade in $T$ and the amount of money satisfying the restrictions imposed by the price controls, so 


$$
\bar{Y}=\left\{y \in B \times S \times L \times \mathbb{R} \mid(\beta(y), \sigma(y), \lambda(y)) \in T \text { and } \underline{p}_{\lambda(y)} \leq \mu(y) \leq \bar{p}_{\lambda(y)}\right\} .
$$

We assume that for every commodity its set of traders has a product structure. Whenever there is a trade in $T$ involving commodity $\ell$ by buyer $b_{1}$ and seller $s_{1}$ as well as a trade involving commodity $\ell$ by buyer $b_{2}$ and seller $s_{2}$, then there is also a trade in $T$ involving commodity $\ell$ by buyer $b_{1}$ and seller $s_{2}$, and a trade in $T$ involving commodity $\ell$ by buyer $b_{2}$ and seller $s_{1}$. Equivalently, for every $\ell \in L$, construct the graph with as nodes the buyers $\beta\left(\bar{Y}_{\ell}\right)$ and the sellers $\sigma\left(\bar{Y}_{\ell}\right)$ and an edge between a buyer $b$ and a seller $s$ if and only if $(b, s, \ell) \in T$. Then, for every commodity $\ell \in L$, this yields a complete bipartite graph if and only if its set of traders has a product structure.

In general equilibrium models, a commodity is defined by its physical characteristics, time, and place of availability. This definition of a commodity is sufficient to guarantee that a given commodity is traded against a single price. Since trade is anonymous, the assumption of a product structure is automatically satisfied. The assumption of traders in a given commodity having a product structure provides a generalization of the standard model to the situation where the feasible trades between a buyer and a seller are explicitly specified and is a sufficient condition to guarantee that a given commodity is traded against a single price.

In models with a set of feasible trades $T$, the assumption of a product structure is without loss of generality, since any form of trading restrictions can be incorporated in the notion of a commodity. For instance, if buyer $b_{1}$ can acquire some product both from seller $s_{1}$ and $s_{2}$, but buyer $b_{2}$ can only purchase this product from $s_{2}$, then we would define two commodities, say $\ell$ and $\ell^{\prime}$. The set $T$ contains trades in commodity $\ell$ by buyer $b_{1}$ and seller $s_{1}$ and by buyer $b_{1}$ and seller $s_{2}$. It also contains a trade in commodity $\ell^{\prime}$ by buyer $b_{2}$ and seller $s_{2}$. In the extreme, we could define as many commodities as there are trades. The resulting notion of competitive equilibrium would then correspond to competitive equilibrium with personalized prices as defined in Hatfield et al. (2013).

When defining equilibrium notions, we require that a commodity is traded against a single price. The less commodities there are, the more demanding this requirement is. On the other hand, the notion of stable outcome does not depend on the definition of a commodity since the contracts that can be written by a pair of agents are not materially affected by this definition. For instance, if we introduce as many commodities as trades in the example of the previous paragraph, then the set of contracts that can be written is isomorphic to the original set. Our equivalence result now implies that as long as the product structure is satisfied, the set of equilibrium allocations is not affected by the definition of a commodity, though the set of supporting prices may.

The commodities that can be traded by an agent $i \in I$ are defined by

$$
L^{i}=\lambda\left(\bar{Y}^{i}\right)=\left\{\ell \in L \mid \exists y \in \bar{Y}^{i} \text { such that } \lambda(y)=\ell\right\} .
$$

We assume that $\cup_{b \in B} L^{b}=\cup_{s \in S} L^{s}=L$. For $\ell \in L$, let $e(\ell)$ be the $\ell$-th unit vector in $\mathbb{R}^{L}$. Then the set of feasible contracts $\bar{Y}$ defines the consumption set $X^{b}$ of a buyer $b \in B$ to be equal to

$$
X^{b}=\mathbb{R} \times\left(\left\{0^{L}\right\} \cup\left\{e(\ell) \mid \ell \in L^{b}\right\}\right),
$$

where $0^{L}$ denotes the zero vector in $\mathbb{R}^{L}$ and corresponds to the case where the buyer does not sign any contract. The unit vector $e(\ell)$ results when buyer $b$ signs a contract involving commodity $\ell$. The first component of a vector $x^{b} \in X^{b}$ denotes the amount of money consumed and is denoted by $x_{0}^{b}$. If buyer $b$ signs a contract involving an amount of money $m$, then $x_{0}^{b}=-m$. If buyer $b$ does not sign a contract, then $x_{0}^{b}=0$. 
The consumption set $X^{s}$ of a seller $s$ is given by

$$
X^{s}=\mathbb{R} \times\left(\left\{0^{L}\right\} \cup\left\{-e(\ell) \mid \ell \in L^{s}\right\}\right),
$$

where we make the usual convention that the supply of commodity $\ell$ by a seller $s$ results in a negative consumption, $x_{\ell}^{s}=-e(\ell)$. If seller $s$ signs a contract involving an amount of money $m$, then $x_{0}^{s}=m$. If seller $s$ does not sign a contract, then $x_{0}^{s}=0$.

We denote $X=\prod_{i \in I} X^{i}$.

The preferences of an agent $i \in I$ are represented by a utility function $u^{i}: X^{i} \rightarrow \mathbb{R}$. The concept of a commodity encompasses all utility relevant information. For a buyer the utility of a commodity does not depend on the agent delivering it, and for a seller the utility of a commodity is independent of the identity of its buyer. This assumption is without loss of generality. Whenever such information is relevant, it can be included in the description of a commodity.

Utility functions are assumed to be continuous and strongly monotonic in $x_{0}$. There are limits to the monetary transfers buyers are willing to make for commodities without price ceilings. For every $b \in B$, for every $\ell \in L^{b}$ such that $\bar{p}_{\ell}=+\infty$, there is $m \in \mathbb{R}$ such that $u^{b}(-m, e(\ell)) \leq u^{b}\left(0,0^{L}\right)$. There are limits to the monetary transfers sellers are willing to make for commodities without price floors. For every $s \in S$, for every $\ell \in L^{s}$ such that $\underline{p}_{\ell}=-\infty$, there is $m \in \mathbb{R}$ such that $u^{s}(m,-e(\ell)) \leq u^{s}\left(0,0^{L}\right)$. Commodities are allowed to be bads, so for a buyer it might be that for some commodity $\ell$ it holds that $u^{b}(0, e(\ell))<u^{b}\left(0,0^{L}\right)$ and for a seller that $u^{s}(0,-e(\ell))>u^{s}\left(0,0^{L}\right)$. Quasi-linear utility functions are a prominent and commonly used example of utility functions satisfying our assumptions.

The primitives of an economy are summarized by $\mathcal{E}=\left(T, p, \bar{p},\left(u^{i}\right)_{i \in I}\right)$.

Although simple, this model includes a number of important special cases.

Example 2.1. Gale and Shapley (1962), marriage problem.

A community consists of $n$ men and $n$ women. Each person ranks those of the oppositive sex in accordance with his or her preferences for a marriage partner. Monetary transfers are not allowed. We define $B$ as the set of men, $S$ as the set of women, and the set of commodities as $L=B \times S$. This is clearly a case with many trading restrictions, since buyers and sellers are both restricted to trade in the commodity carrying their label, $T=\{(b, s, \ell) \in B \times S \times L \mid \ell=(b, s)\}$. A commodity corresponds to a contract between a man and a woman. The absence of monetary transfers is achieved by setting $\underline{p}=\bar{p}=0^{L}$. Utility functions are now specified in accordance with each person's ranking of the partners of opposite sex, with the utility of remaining single strictly below the lowest utility assigned to a partner when $x_{0}=0$. Since monetary transfers are not possible, the specification of the utility function is immaterial for other values of $x_{0}$. Since there are as many commodities as there are trades, $T$ trivially has a product structure.

Example 2.2. Shapley and Shubik (1971), assignment model.

There are $m$ homeowners in the real estate market and $n$ prospective purchasers. Homeowner $s$ values his house at $c_{s}$ dollars, while purchaser $b$ values the same house at $h_{b s}$ dollars. There are no restrictions on monetary transfers. We define $B$ as the set of prospective purchasers, $S$ as the set of homeowners, and the set of commodities as $L=S$. A commodity corresponds to a house. Again, there are many trading restrictions, since a seller is restricted to trade in the house bearing his label, $T=\{(b, s, \ell) \in B \times S \times L \mid \ell=s\}$. There are no restrictions on monetary transfers, so for every $\ell \in L$ we set $\underline{p}_{\ell}=-\infty$ and $\bar{p}_{\ell}=+\infty$. The utility of a buyer $b \in B$ is given by 


$$
u^{b}(x)=x_{0}+\sum_{s \in S} h_{b s} x_{s} .
$$

The utility of a seller $s \in S$ is equal to

$$
u^{s}(x)=x_{0}+c_{s} x_{s} .
$$

It is easily verified that this application satisfies all the assumptions made before.

Notice that a commodity depends on both the buyer and the seller in the marriage problem of Example 2.1, but only on the seller in the assignment model of Example 2.2.

Example 2.3. Hatfield et al. (2012), quality competition in the presence of price controls.

Several authors have argued that the presence of price controls affects the commodity which is traded on the market. For instance, Leffler (1982) argues that price ceilings give rise to the supply of inefficiently low quality. Other authors, like Feldstein (1973), have studied the effect of minimum wages on job quality, and Agell and Lommerud (1997) analyze the effects of minimum wages on worker's skill formation. To study the effects of price controls on traded quality, Hatfield et al. (2012) develop a model of quality competition.

The set of commodities is $L=\{1, \ldots, \bar{\ell}\}$. Commodities have universally agreed upon qualities, with the quality of commodity $\ell$ exceeding the quality of commodity $k$ if and only if $\ell \geq k$. Commodities are therefore referred to as qualities. There is a price control that specifies a common price floor $f$ for all qualities: for every $\ell \in L, \underline{p}_{\ell}=f$. There are no price ceilings, so for every $\ell \in L, \bar{p}_{\ell}=+\infty$. The value for buyer $b$ of procuring any quality is given by $v^{b} \geq 0$, and $v_{\ell}$ denotes the additional utility derived from procuring quality $\ell$. To obtain a unique utility representation, it is assumed that $v_{1}=0$. For $\ell=2, \ldots, \bar{\ell}$, we define $\Delta v_{\ell}=v_{\ell}-v_{\ell-1}$ as the difference in utility between quality $\ell$ and quality $\ell-1$. It is assumed that $\Delta v_{\ell}$ is positive. The utility of a buyer $b \in B$ is then given by

$$
u^{b}(x)=x_{0}+v^{b} \sum_{\ell \in L} x_{\ell}+\sum_{\ell \in L} v_{\ell} x_{\ell} .
$$

Sellers produce at most one quality. The cost for seller $s$ of producing any quality is given by $c^{s} \geq$ 0 , and $c_{\ell}$ denotes the additional cost of producing commodity $\ell$. To obtain a unique representation of the costs, it is assumed that $c_{1}=0$. For $\ell=2, \ldots, \bar{\ell}$, we define $\Delta c_{\ell}=c_{\ell}-c_{\ell-1}$ as the increase in costs to go from quality $\ell-1$ to quality $\ell$. It is assumed that $\Delta c_{\ell}$ is positive. The utility of a seller $s \in S$ is given by the profit

$$
u^{s}(x)=x_{0}+c^{s} \sum_{\ell \in L} x_{\ell}+\sum_{\ell \in L} c_{\ell} x_{\ell} .
$$

\section{Stable outcomes and Drèze equilibria}

In this section, we present the concept of stable outcome from matching theory and the concept of Drèze equilibrium from general equilibrium theory.

A set of contracts $A \subset \bar{Y}$ is an outcome if each agent is involved in at most one contract, so the cardinality of every set $A^{i}$ is at most one. The set of all outcomes is denoted by the collection $\mathcal{A}=\left\{A \subset \bar{Y} \mid\right.$ for every $\left.i \in I,\left|A^{i}\right| \leq 1\right\}$. In particular, it holds that $\emptyset \in \mathcal{A}$. For $i \in I$, the collection $\mathcal{A}^{i}$ is defined as the collection containing the empty set together with all sets consisting of a single contract that involves agent $i$. This set corresponds to the set of possible choices for 
agent $i$. More formally, $\mathcal{A}^{i}=\{A \in \mathcal{A} \mid$ for every $y \in A, i \in \iota(y)\}$. An outcome $A$ generates the unique allocation $\xi(A) \in X$ defined as follows. For every $b \in B$, if $A^{b}=\emptyset$, then $\xi^{b}(A)=\left(0,0^{L}\right)$; otherwise, let $y$ be the unique contract in $A^{b}$ and define $\xi^{b}(A)=(-\mu(y), e(\lambda(y)))$. For every $s \in S$, if $A^{s}=\emptyset$, then $\xi^{s}(A)=\left(0,0^{L}\right)$; otherwise, let $y$ be the unique contract in $A^{s}$ and define $\xi^{s}(A)=(\mu(y),-e(\lambda(y)))$. Since every contract specifies the delivery of a commodity from a seller to a buyer in exchange for a particular quantity of money, it holds that

$$
\sum_{i \in I} \xi^{i}(A)=0
$$

The utility of agent $i \in I$ derived from outcome $A \in \mathcal{A}$ is given by

$$
U^{i}(A)=u^{i}\left(\xi^{i}(A)\right) .
$$

The choice relation $C^{i}$ of agent $i \in I$ is defined by

$$
C^{i}(Y)=\arg \max _{\left\{A^{i} \in \mathcal{A}^{i} \mid A^{i} \subset Y^{i}\right\}} U^{i}\left(A^{i}\right), \quad Y \subset \bar{Y} .
$$

The set $C^{i}(Y)$ contains all the alternatives $A^{i}$ in the set of possible choices $\mathcal{A}^{i}$ of agent $i$ that are a subset of $Y^{i}$ and that maximize his utility. Since in general $C^{i}$ might be empty-valued, we use the term choice relation rather than choice correspondence. In the sequel, we will apply $C^{i}$ to finite sets $Y$, which guarantees that $C^{i}$ is not empty-valued. The next definition reformulates the stability concept as defined in Roth and Sotomayor (1990) to make it compatible with our set-up.

Definition 3.1. An outcome $A \in \mathcal{A}$ is stable if:

1. For every $i \in I, A^{i} \in C^{i}(A)$.

2. For every $z=(b, s, \ell, m) \in \bar{Y}$ it holds that $U^{b}(\{z\}) \leq U^{b}(A)$ or $U^{s}(\{z\}) \leq U^{s}(A)$.

A stable outcome $A$ involves only contracts in the set of feasible contracts $\bar{Y}$ and at most one contract for each agent. The first condition in Definition 3.1 corresponds to individual rationality. Every agent $i$ should weakly prefer his current contract to staying inactive. The second condition requires absence of blocking by a two-agent coalition.

We now develop a notion of competitive equilibrium for the economy $\mathcal{E}=\left(T, p, \bar{p},\left(u^{i}\right)_{i \in I}\right)$ as presented in Section 2. As is usual, see, e.g., Gul and Stacchetti (1999) and Sun and Yang (2006), at such an equilibrium each commodity is traded against a single price. The initial endowment of every agent $i \in I$ is equal to $\left(0,0^{L}\right) \in X^{i}$.

For economies in which all commodities are perfectly divisible, where consumption sets are equal to $\mathbb{R}_{+} \times \mathbb{R}_{+}^{L}$, there is an extensive literature on competitive equilibrium under price controls, starting with seminal contributions by Bénassy (1975), Drèze (1975), and Younès (1975). Herings (1996) provides an overview of this stream in the literature. Here, we follow the approach as developed in Drèze (1975) and extend it to the economies with indivisible commodities of Section 2.

In a competitive analysis of price controls, there is a price vector $p \in\{1\} \times \mathbb{R}^{L}$ against which trade in commodities takes place. In the presence of a price floor $p$ and a price ceiling $\bar{p}$, it should hold that $\underline{p}_{\ell} \leq p_{\ell} \leq \bar{p}_{\ell}$ for every $\ell \in L$. A buyer $b \in B$ chooses a utility maximizing element $x^{b} \in X^{b}$ subject to the budget constraint $p x^{b} \leq 0$. Similarly, a seller $s \in S$ chooses a utility maximizing element $x^{s} \in X^{s}$ subject to the budget constraint $p x^{s} \leq 0$. Moreover, buyers and sellers may face rationing constraints to be explained below. 
In case the price ceiling $\bar{p}_{\ell}$ is binding for some commodity $\ell$, its market may be cleared by quantity adjustments. Since prices are upward rigid, quantity adjustments are on the buyers' side. At a price equal to $\bar{p}_{\ell}$, a buyer might be willing to buy commodity $\ell$, but there is no seller willing to supply to him at that price. The price ceiling prevents the price from going up. Under such circumstances, a buyer faces demand rationing of commodity $\ell$.

Let $Q \subset\left\{(b, \ell) \in B \times L \mid \ell \in L^{b}\right\}$ be the set of demand rationing constraints. The collection of all such sets is denoted by $\mathcal{Q}$, so

$$
\mathcal{Q}=\left\{Q \in 2^{B \times L} \mid \text { for every }(b, \ell) \in Q, \ell \in L^{b}\right\} .
$$

If $(b, \ell) \in Q$, then buyer $b$ is rationed in his demand of commodity $\ell$, and when maximizing his utility, he takes into account the constraint $x_{\ell}^{b}=0$. The set $Q$ is determined endogenously in an equilibrium and could be equal to the empty set in which case no demand rationing is present. For $b \in B$, we define $Q^{b}=\{\ell \in L \mid(b, \ell) \in Q\}$ and for $\ell \in L$, we define $Q_{\ell}=\{b \in B \mid(b, \ell) \in Q\}$. The set $Q^{b}$ yields the commodities in which buyer $b$ is rationed in his demand. The set $Q_{\ell}$ contains the buyers which are rationed in their demand of commodity $\ell$.

In case the price floor $\underline{p}_{\ell}$ is binding for some commodity $\ell$, its market may be cleared by quantity adjustments. Since prices are downwards rigid, the quantity adjustments are on the sellers' side. Indeed, a seller may not face any demand for commodity $\ell$ when the price is $\underline{p}_{\ell}$, although he is willing to sell commodity $\ell$ against price $\underline{p}_{\ell}$. Moreover, the price floor prevents the price from going down. Under such circumstances, the seller faces supply rationing of commodity $\ell$.

Let $R \subset\left\{(s, \ell) \in S \times L \mid \ell \in L^{s}\right\}$ be the set of supply rationing constraints. The collection of all such sets is denoted by $\mathcal{R}$, so

$$
\mathcal{R}=\left\{R \in 2^{S \times L} \mid \text { for every }(s, \ell) \in R, \ell \in L^{s}\right\} .
$$

If $(s, \ell) \in R$, then seller $s$ is rationed in his supply of commodity $\ell$, and when maximizing his utility, he takes into account the constraint $x_{\ell}^{S}=0$. The set $R$ is determined endogenously in an equilibrium and could be equal to the empty set in which case no supply rationing is present. For $s \in S$, we define $R^{s}=\{\ell \in L \mid(s, \ell) \in R\}$ and for $\ell \in L$, we define $R_{\ell}=\{s \in S \mid(s, \ell) \in R\}$. The set $R^{s}$ yields the commodities in which seller $s$ is rationed in his supply. The set $R_{\ell}$ contains the sellers which are rationed in their supply of commodity $\ell$.

Given a price vector $p \in\{1\} \times \mathbb{R}^{L}$ and a set of rationing constraints $Q^{b} \subset L^{b}$, the budget set of a buyer $b \in B$ is given by

$$
\gamma^{b}\left(p, Q^{b}\right)=\left\{x^{b} \in X^{b} \mid p x^{b} \leq 0, \text { for every } \ell \in Q^{b}, x_{\ell}^{b}=0\right\} .
$$

The rationing constraints of buyers different from $b$ are irrelevant for the budget set of buyer $b$ and are therefore suppressed in the definition of $\gamma^{b}$. The demand correspondence $\delta^{b}$ of buyer $b$ is defined by

$$
\delta^{b}\left(p, Q^{b}\right)=\arg \max _{x^{b} \in \gamma^{b}\left(p, Q^{b}\right)} u^{b}\left(x^{b}\right) .
$$

The budget set $\gamma^{b}\left(p, Q^{b}\right)$ is non-empty as it contains $\left(0,0^{L}\right)$ as an element. It follows that $\delta^{b}\left(p, Q^{b}\right)$ is non-empty, since monotonicity of the utility function in money implies that the budget constraint can be defined with equality, and the maximization therefore takes place over a non-empty finite set of elements.

Given a price vector $p \in\{1\} \times \mathbb{R}^{L}$ and a set of rationing constraints $R^{s} \subset L^{s}$, the budget set of a seller $s \in S$ is given by 


$$
\gamma^{s}\left(p, R^{s}\right)=\left\{x^{s} \in X^{s} \mid p x^{s} \leq 0, \text { for every } \ell \in R^{s}, x_{\ell}^{s}=0\right\}
$$

The rationing constraints of sellers different from $s$ are irrelevant for the budget set of seller $s$ and are therefore omitted in the definition of $\gamma^{s}$. The demand correspondence $\delta^{s}$ of seller $s$ is defined by

$$
\delta^{s}\left(p, R^{s}\right)=\arg \max _{x^{s} \in \gamma^{s}\left(p, R^{s}\right)} u^{s}\left(x^{s}\right)
$$

Using the same argument as before, it follows that $\delta^{s}\left(p, R^{s}\right)$ is non-empty.

Definition 3.2. A Drèze equilibrium of the economy $\mathcal{E}=\left(T, \underline{p}, \bar{p},\left(u^{i}\right)_{i \in I}\right)$ is an element $\left(p^{*}, Q^{*}, R^{*}, x^{*}\right) \in\left(\{1\} \times \mathbb{R}^{L}\right) \times \mathcal{Q} \times \mathcal{R} \times X$ such that:

1. For every $b \in B, x^{* b} \in \delta^{b}\left(p^{*}, Q^{* b}\right)$.

2. For every $s \in S, x^{* s} \in \delta^{s}\left(p^{*}, R^{* S}\right)$.

3. $\sum_{i \in I} x^{* i}=\left(0,0^{L}\right)$.

4. For every $\ell \in L, \underline{p}_{\ell} \leq p_{\ell}^{*} \leq \bar{p}_{\ell}$.

5. For every $\ell \in L$, if $p_{\ell}^{*}<\bar{p}_{\ell}$, then $Q_{\ell}^{*}=\emptyset$.

6. For every $\ell \in L$, if $p_{\ell}^{*}>\underline{p}_{\ell}$, then $R_{\ell}^{*}=\emptyset$.

7. For every $\ell \in L, Q_{\ell}^{*}=\emptyset$ or $R_{\ell}^{*}=\emptyset$.

The first two conditions in Definition 3.2 reflect the standard optimizing behavior of the buyers and the sellers. Buyers and sellers need only know the given prices and their individual rationing scheme and need not consider the other individuals in making their choices. The third condition expresses market clearing. The fourth condition rules out exchange against prices violating the price controls. Condition 5 states that whenever the price of a commodity $\ell$ is upwards flexible, i.e. strictly below the price ceiling $\bar{p}_{\ell}$, then there is no rationing of the buyers. Similarly, Condition 6 states that whenever the price of a commodity $\ell$ is downwards flexible, i.e. strictly above the price floor $\underline{p}_{\ell}$, then there is no rationing of the sellers. Condition 7 expresses that markets are transparent. There is no simultaneous rationing on the supply and on the demand of a given commodity $\ell$.

When we apply Definition 3.2 to the case with only one commodity, the analysis reduces to the standard textbook analysis of the effects of price floors and price ceilings based on supply and demand curves.

In the absence of price controls, so when, for every $\ell \in L, p_{\ell}=-\infty$ and $\bar{p}_{\ell}=+\infty$, Definition 3.2 reduces to the standard definition of a Walrasian equilibrium. It follows immediately from Conditions 5 and 6 that $Q^{*}=R^{*}=\emptyset$, so Condition 7 is automatically satisfied. Condition 4 becomes void. What remains are the two standard Conditions 1 and 2, expressing optimization without rationing constraints, and the market clearing Condition 3.

For the assignment model of Example 2.2 with price controls, Talman and Yang (2008) define the concept of constrained Walrasian equilibrium. It is not difficult to show that the application of the Drèze equilibrium of Definition 3.2 to that model coincides with the constrained Walrasian equilibrium. Andersson and Svensson (2014) consider the assignment model of Example 2.2 with price controls. They propose the concept of a rationing price equilibrium. The rationing price equilibrium is a Drèze equilibrium, but not vice versa. 
Table 1

Valuations and costs in the economy of Example 2.3 with $B=\left\{b_{1}, b_{2}, b_{3}\right\}, S=\left\{s_{1}, s_{2}, s_{3}\right\}$, and $L=\{1,2,3\}$.

\begin{tabular}{|l|lll|}
\hline & 1 & 2 & 3 \\
\hline$b_{1}$ & 7 & 8 & 9 \\
$b_{2}$ & 6 & 7 & 8 \\
$b_{3}$ & 2 & 3 & 4 \\
\hline
\end{tabular}

\begin{tabular}{|l|lll|}
\hline & 1 & 2 & 3 \\
\hline$s_{1}$ & 1 & 3 & 6 \\
$s_{2}$ & 2 & 4 & 7 \\
$s_{3}$ & 4 & 6 & 9 \\
\hline
\end{tabular}

\section{An illustration}

To illustrate the usefulness of the model, the concepts of stable outcome and Drèze equilibrium, and the richness of allocations that result, we now examine the model of Example 2.3 in a bit more detail. Consider, for instance, an economy with three buyers, three sellers, and three qualities, where valuations and costs are as depicted in Table 1.

First, consider the case without price floors. It can be easily verified that every stable outcome $A$ involves two contracts, $\beta(A)=\left\{b_{1}, b_{2}\right\}, \sigma(A)=\left\{s_{1}, s_{2}\right\}$, only quality 1 is traded, $\lambda(A)=\{1\}$, and every contract in $A$ trades at a price $m$ in between 2 and 4. Every stable outcome maximizes total surplus.

Now let $\left(p^{*}, Q^{*}, R^{*}, x^{*}\right)$ be a Drèze equilibrium. Since there are neither price floors nor price ceilings, it holds that $Q^{*}=R^{*}=\emptyset$, and the concept of a Drèze equilibrium reduces to a Walrasian equilibrium. Suppose there is trade in quality 2 . Then it should hold that $p_{2}^{*}<p_{1}^{*}+1$, since otherwise there is no demand for it. At the same time, it should hold that $p_{2}^{*}>p_{1}^{*}+2$, since otherwise there is no supply of quality 2 . It follows that there is no trade in quality 2 at a Drèze equilibrium and by a similar argument it can be shown that there is no trade in quality 3 , so for every $i \in I$, it holds that $x_{2}^{* i}=x_{3}^{* i}=0$, whereas $p_{1}^{*}+1 \leq p_{2}^{*} \leq p_{1}^{*}+2$ and $p_{1}^{*}+2 \leq p_{3}^{*} \leq p_{1}^{*}+5$. Now it is easily verified that $2 \leq p_{1}^{*} \leq 4, x_{1}^{* b_{1}}=x_{1}^{* b_{2}}=1$, and $x_{1}^{* s_{1}}=x_{1}^{* s_{2}}=-1$. We also have that $p_{0}^{*}=1, x_{0}^{* b_{1}}=x_{0}^{* b_{2}}=-p_{1}^{*}$, and $x_{0}^{* s_{1}}=x_{0}^{* s_{2}}=p_{1}^{*}$. Finally, it holds that $x^{* b_{3}}=x^{* s_{3}}=$ $\left(0,0^{L}\right)$. The allocations that occur in a Drèze equilibrium are exactly the same as those in a stable outcome.

Now consider a price floor $f \in[2,4]$. All of a sudden, Pareto inefficient stable outcomes on top of the surplus maximizing stable outcomes of the previous paragraph emerge. Consider, for instance, the Pareto inefficient outcome $A^{\prime}=\left\{\left(b_{1}, s_{1}, 2, f+1\right),\left(b_{2}, s_{2}, 1, f\right)\right\}$, where buyer $b_{1}$ trades quality 2 with seller $s_{1}$ against the price $f+1$ and buyer $b_{2}$ trades quality 1 with seller $s_{2}$ against the price $f$.

We verify next that $A^{\prime}$ is stable. Obviously, every contract in $A^{\prime}$ obeys individual rationality. Suppose there is a contract $z=(b, s, \ell, m)$ which gives both $b$ and $s$ strictly higher utility. It holds that $U^{b_{1}}\left(A^{\prime}\right)=8-f-1=7-f$, so to give $b_{1}$ strictly higher utility, it should hold that $\ell=1$ and $m<f$, or $\ell=2$ and $m<f+1$, or $\ell=3$ and $m<f+2$. The first case is not possible in the presence of a price floor equal to $f$. The latter two cases are not possible since the utility for $s$ would be lower than $U^{s}\left(A^{\prime}\right)$, irrespective of the choice for $s$. It follows that $b \neq b_{1}$.

The same argument can be used to show that $b \neq b_{2}$.

Buyer $b_{3}$ cannot get a positive utility by consuming quality 1 against price $f$, and no seller can produce quality 2 against a cost lower than $v^{b_{3}}+v_{2}=3$ or quality 3 against a cost lower than $v^{b_{3}}+v_{3}=4$, so $b \neq b_{3}$. This concludes the proof that outcome $A^{\prime}$ is stable. This example is striking because it shows how Pareto inefficient stable outcomes can exist in the presence of 
price floors that are sufficiently low to be compatible with stable outcomes in the absence of price floors.

We now construct a Drèze equilibrium $\left(p^{*}, Q^{*}, R^{*}, x^{*}\right)$ such that $x^{*}=\xi\left(A^{\prime}\right)$. We clearly need that $p_{1}^{*}=f$ and $p_{2}^{*}=f+1$. Let $p_{3}^{*}$ be such that $f+2 \leq p_{3}^{*} \leq f+5$. Since there are no price ceilings in this model, it holds by Condition 5 of Definition 3.2 that $Q^{*}=\emptyset$. Since the prices of qualities 2 and 3 exceed the price floor, there is no supply rationing of these qualities by Condition 6 of Definition 3.2 and $R_{2}^{*}=R_{3}^{*}=\emptyset$. At the given prices and rationing schemes, it holds that $x^{* b_{1}}=(-f-1,0,1,0) \in \delta^{b_{1}}\left(p^{*}, Q^{* b_{1}}\right), x^{* b_{2}}=(-f, 1,0,0) \in \delta^{b_{2}}\left(p^{*}, Q^{* b_{2}}\right)$, and $x^{* b_{3}}=\left(0,0^{L}\right) \in \delta^{b_{3}}\left(p^{*}, Q^{* b_{3}}\right)$. Irrespective of the choice for $R_{1}^{*}$, it holds that $0 \in \delta^{s_{3}}\left(p^{*}, R^{* s_{3}}\right)$. In the absence of rationing, both seller $s_{1}$ and seller $s_{2}$ would strictly prefer to produce quality 1 , which would result in excess supply of quality 1 . Due to the price floor, there is no possibility for the price of quality 1 to drop, and one of the two sellers gets rationed on his supply. To achieve the allocation $\xi\left(A^{\prime}\right)$, this will be seller $s_{1}: R_{1}^{*}=\left\{s_{1}\right\}$. We now have $x^{* s_{1}}=(f+1,0,-1,0) \in$ $\delta^{s_{1}}\left(p^{*}, R^{* s_{1}}\right)$ and $x^{* s_{2}}=(f,-1,0,0) \in \delta^{s_{2}}\left(p^{*}, R^{* s_{2}}\right)$. All the conditions of Definition 3.2 are satisfied and the Drèze equilibrium allocation is equal to $\xi\left(A^{\prime}\right)$.

\section{Existence of stable outcomes}

In this section, we present an adjustment process in the spirit of Gale and Shapley (1962), Crawford and Knoer (1981), Kelso and Crawford (1982), Demange et al. (1986), and Hatfield and Milgrom (2005) that ends with a stable outcome. The reason for presenting this adjustment process is twofold. First, the existence of a stable outcome in our model does not follow from existing results in the literature. For instance, Kelso and Crawford (1982) do not allow for price rigidities or contracts, whereas Hatfield and Milgrom (2005) rule out indifferences. Secondly, and more important, when applied to the special cases of our model as discussed in Section 2, the adjustment process coincides with known adjustment processes in the literature. The adjustment process therefore unifies the adjustment processes as used in the literature in exactly the same way as the model unifies the existing models as discussed in Section 2.

We first reduce the set of possible contracts to a finite subset of $\bar{Y}$. Consider some $t=$ $(b, s, \ell) \in T$. Without loss of generality, we assume that there is $m \in\left[\underline{p}_{\ell}, \bar{p}_{\ell}\right] \cap \mathbb{R}$ such that $u^{b}(-m, e(\ell)) \geq u^{b}\left(0,0^{L}\right)$ and $u^{s}(m,-e(\ell)) \geq u^{s}\left(0,0^{L}\right)$. Otherwise, a trade can never be part of a stable outcome by individual rationality and can be dropped from $T$. Contracts $(t, m) \in \bar{Y}$ that are individually rational for both the buyer and the seller are such that $m$ belongs to a non-empty interval $\left[\underline{m}_{t}, \bar{m}_{t}\right]$, where $\underline{m}_{t}$ and $\bar{m}_{t}$ are both finite, $\underline{m}_{t} \geq \underline{p}_{\ell}$, and $\bar{m}_{t} \leq \bar{p}_{\ell}$. The details of the argument are provided in the appendix, Lemma A.1.

Next, we choose some $\varepsilon>0$ and define the finite set of contracts $\bar{Y}^{\varepsilon}$ by

$$
\bar{Y}^{\varepsilon}=\left\{(t, m) \in \bar{Y} \mid \exists k \in \mathbb{N}_{0} \text { such that } m=\min \left\{\underline{m}_{t}+k \varepsilon, \bar{m}_{t}\right\}\right\} .
$$

In this way, only contracts $(t, m)$ are considered where the difference between $m$ and $\underline{m}_{t}$ is an integer multiple of $\varepsilon$ or $m=\bar{m}_{t}$. Moreover, this definition achieves that $m$ belongs to the interval $\left[\underline{m}_{t}, \bar{m}_{t}\right]$.

The adjustment process is now defined as follows.

\section{Definition 5.1 (Adjustment Process).}

Step 1: Initially, the set of permitted contracts $Y$ is equal to $\bar{Y}^{\varepsilon}$ and the set of tentatively accepted contracts $A$ is equal to $\emptyset$. 
Step 2: Every buyer $b \in B \backslash \beta(A)$ proposes an arbitrarily chosen element of $C^{b}(Y)$. Let $Z$ be the union of the set of contracts as chosen by $b \in B \backslash \beta(A)$ and the set $A$.

Step 3: Every seller $s \in \sigma(Z)$ tentatively accepts one arbitrarily chosen element of $C^{s}(Z)$. Let $A$ be the set of contracts as tentatively accepted by $s \in \sigma(Z)$.

Step 4: The process stops if $A=Z$. In that case the contracts in $A$ are permanently accepted. Otherwise, the process returns to Step 2 with set of permitted contracts $Y \backslash(Z \backslash A)$.

Since the set $Y$ is finite and a buyer can always choose $\emptyset$, it holds that the set $C^{b}(Y)$ in Step 2 is non-empty. The same remark applies to the set $C^{S}(Z)$ in Step 3.

It can be easily verified that for the marriage problem of Example 2.1, the adjustment process coincides with the deferred acceptance algorithm of Gale and Shapley (1962). For the assignment model of Example 2.2, the adjustment process coincides with the approximate auction mechanism as discussed in Section V of Demange et al. (1986). In the case without ties, the adjustment process coincides with the generalized Gale-Shapley algorithm of Hatfield and Milgrom (2005).

A stable outcome $A$ for the set of feasible contracts $\bar{Y}^{\varepsilon}$ is defined as in Definition 3.1 with $\bar{Y}$ replaced by $\bar{Y}^{\varepsilon}$. Theorem A.2 in the appendix shows that the adjustment process of Definition 5.1 terminates in a finite number of steps with a set $A$ of permanently accepted contracts, which is a stable outcome for the set of feasible contracts $\bar{Y}^{\varepsilon}$. A limit argument can now be used to demonstrate the existence of a stable outcome in the model without a smallest monetary unit.

Theorem 5.2. Consider an economy $\mathcal{E}=\left(T, \underline{p}, \bar{p},\left(u^{i}\right)_{i \in I}\right)$. A stable outcome exists.

Proof. See Appendix.

\section{Equivalence of stable outcomes and Drèze equilibria}

Shapley and Shubik (1971) consider the assignment game and prove that the set of stable payoffs coincides with the set of competitive equilibrium payoffs. This relationship between stable outcomes and competitive equilibria has been substantially generalized, in the direction with general, not necessarily quasi-linear, utility functions by Kaneko (1982) and Quinzii (1984) and in the direction of set-ups with many-to-many matching or even trading networks as demonstrated by Hatfield et al. (2013). These results are invariably derived in a setting with unlimited monetary transfers, or, equivalently, absence of price controls. In this section, we obtain a fully general equivalence between stable outcomes and Drèze equilibria. This equivalence boils down to the equivalence with competitive equilibria in the absence of price controls, presents a completely new result for cases with no monetary transfers like the marriage problem, and also deals with all intermediate cases like wage or rent controls.

The concepts of stable outcome and Drèze equilibrium are not directly comparable. On the one hand, a stable outcome provides more information, as it is specified who trades with whom. On the other hand, in a Drèze equilibrium also the prices of commodities that are not traded are specified and a Drèze equilibrium specifies explicit rationing constraints.

Although it is not possible to directly compare a stable outcome to a Drèze equilibrium, we can compare the resulting allocations. In fact, this is all that matters from a welfare point of view.

Consider an economy $\mathcal{E}=\left(T, p, \bar{p},\left(u^{i}\right)_{i \in I}\right)$. The set of allocations corresponding to stable outcomes is $X^{\text {so }}$,

$$
X^{\text {so }}=\{x \in X \mid \text { there is a stable outcome } A \text { such that } \xi(A)=x\} .
$$


The set of Drèze equilibrium allocations is $X^{\mathrm{D}}$,

$$
X^{\mathrm{D}}=\left\{x^{*} \in X \mid \text { there is }\left(p^{*}, Q^{*}, R^{*}\right) \text { such that }\left(p^{*}, Q^{*}, R^{*}, x^{*}\right) \text { is a Drèze equilibrium }\right\} .
$$

The next result shows that if $A$ is a stable outcome, then there is a Drèze equilibrium with allocation $\xi(A)$.

Theorem 6.1. Consider an economy $\mathcal{E}=\left(T, \underline{p}, \bar{p},\left(u^{i}\right)_{i \in I}\right)$. It holds that $X^{\text {so }} \subset X^{\mathrm{D}}$.

Proof. See Appendix.

Consider some stable outcome $A$. Step 1 in the proof of Theorem 6.1 shows that each commodity in $\lambda(A)$ trades at a single price. Indeed, for every $y, y^{\prime} \in A$ such that $\lambda(y)=\lambda\left(y^{\prime}\right)$ it holds that $\mu(y)=\mu\left(y^{\prime}\right)$. Intuitively, whenever two contracts involve different amounts of money, the buyer paying the high amount wants to match with the seller receiving the low amount, and write a contract with an amount of money somewhere in the middle. Since $T$ has a product structure, such a match is feasible. This makes it possible to use the stable outcome $A$ in the construction of a Drèze equilibrium, where a commodity trades against a single price by definition. The price of a commodity $\ell \in \lambda(A)$ is denoted by $p_{\ell}^{*}$.

For $\ell \in L$ and $b \in \beta\left(\bar{Y}_{\ell}\right)$, the extended real number $r_{\ell}^{b}$ denotes the reservation value of commodity $\ell$ for buyer $b$. By definition, it is equal to the value of $m$ for which $u^{b}(-m, e(\ell))=$ $u^{b}\left(x^{* b}\right)$, where $x^{* b}$ is the consumption bundle of buyer $b$ as induced by the stable outcome $A .{ }^{1}$ The highest reservation value among all buyers in $\beta\left(\bar{Y}_{\ell}\right)$ is denoted by $r_{\ell}^{B}$. Similarly, for $\ell \in L$ and $s \in \sigma\left(\bar{Y}_{\ell}\right), r_{\ell}^{s}$ denotes the reservation value of commodity $\ell$ for seller $s$, so $u^{s}\left(r_{\ell}^{s},-e(\ell)\right)=$ $u^{s}\left(x^{* S}\right)$, and $r_{\ell}^{S}$ denotes the lowest reservation value among all sellers in $\sigma\left(\bar{Y}_{\ell}\right)$.

In Step 3 of the proof of Theorem 6.1 it is shown that for any traded commodity $\ell \in \lambda(A)$ at least one of three cases holds. The first possibility is that $p_{\ell}^{*}=\underline{p}_{\ell}$ and $r_{\ell}^{S} \leq \underline{p}_{\ell}=r_{\ell}^{B}$. In this case, a seller $s$ may have a reservation value $r_{\ell}^{s}<\underline{p}_{\ell}$, but the price floor $\underline{p}_{\ell}$ prevents a price decrease and the seller is rationed on his supply of commodity $\ell$. Since none of the buyers $b$ has a reservation value $r_{\ell}^{b}>p_{\ell}^{*}$, there is no need for rationing buyers on their demand of commodity $\ell$.

The second possibility is that $\underline{p}_{\ell} \leq p_{\ell}^{*} \leq \bar{p}_{\ell}$ and $r_{\ell}^{S}=p_{\ell}^{*}=r_{\ell}^{B}$. This case corresponds to a market without effective price controls and absence of rationing. It will always occur when the price floor $\underline{p}_{\ell}$ is equal to $-\infty$ and the price ceiling $\bar{p}_{\ell}$ is equal to $+\infty$.

The third possibility of Step 3 parallels the first one with the role of buyers replaced by sellers. It holds that $p_{\ell}^{*}=\bar{p}_{\ell}$ and $r_{\ell}^{S}=\bar{p}_{\ell} \leq r_{\ell}^{B}$. In this case, a buyer $b$ may have a reservation value $r_{\ell}^{b}>\bar{p}_{\ell}$, but the price ceiling $\bar{p}_{\ell}$ prevents a price increase and the buyer is rationed on his demand of commodity $\ell$. Since none of the sellers $s$ has a reservation value $r_{\ell}^{s}<p_{\ell}^{*}$, there is no need for rationing sellers on their supply of commodity $\ell$.

In degenerate cases, the first and the third possibility can occur simultaneously. In this case the price floor must be equal to the price ceiling, the lowest reservation value among sellers, and the highest reservation value among buyers. The behavior of the market resembles the one of the second possibility. There are no effective price controls and there is absence of rationing.

In Step 4 of the proof of Theorem 6.1 it is shown that for any non-traded commodity $\ell \in$ $L \backslash \lambda(A)$ at least one of three cases holds. The first possibility is that $r_{\ell}^{B} \leq \underline{p}_{\ell}$. In that case, the

\footnotetext{
${ }^{1}$ In the proof, we also deal carefully with the cases where $u^{b}(-m, e(\ell))<u^{b}\left(x^{* b}\right)$ for all $m \in \mathbb{R}$ and $u^{b}(-m, e(\ell))>$ $u^{b}\left(x^{* b}\right)$ for all $m \in \mathbb{R}$.
} 
price floor is so high that all potential trade in commodity $\ell$ is killed off. The lowest reservation value among sellers of commodity $\ell$ may be higher or lower than $r_{\ell}^{B}$. In case it is lower, welfare gains could be achieved by contracts among such a seller and a buyer with a strictly higher reservation value. Since $r_{\ell}^{S}$ can be strictly below the price floor $\underline{p}_{\ell}$, supply rationing of sellers may occur in equilibrium. Since $r_{\ell}^{B} \leq \underline{p}_{\ell}$, there will be no simultaneous rationing of the demand of buyers. The equilibrium price $p_{\ell}^{*}$ of such a non-traded commodity can be set equal to $\underline{p}_{\ell}$ if $\underline{p}_{\ell}>-\infty$. If $\underline{p}_{\ell}=-\infty$, and therefore $r_{\ell}^{B}=-\infty$, so none of the buyers have an interest to acquire commodity $\ell$ against any price, $p_{\ell}^{*}$ can be set equal to any value less than or equal to the price ceiling $\bar{p}_{\ell}$ and the lowest reservation value among all sellers $r_{\ell}^{S}$.

The second possibility concerns the case where $r_{\ell}^{B}<r_{\ell}^{S}, \underline{p}_{\ell}<r_{\ell}^{B}$, and $r_{\ell}^{S}<\bar{p}_{\ell}$. The market for such a commodity resembles a market without price controls and does not involve any rationing. Commodity $\ell$ does simply not admit a profitable trade among a buyer and a seller in this case. The equilibrium price of such a commodity can be any value in between $r_{\ell}^{B}$ and $r_{\ell}^{S}$.

The third possibility of Step 4 is the case where $\bar{p}_{\ell} \leq r_{\ell}^{S}$. This is the analogue of the first possibility with buyers replaced by sellers. The price ceiling is so low that all potential supply of commodity $\ell$ is wiped out. If the highest reservation value among buyers exceeds $\bar{p}_{\ell}$, then demand rationing of such buyers occurs. The equilibrium price of such a commodity is taken equal to $\bar{p}_{\ell}$ if $\bar{p}_{\ell}<+\infty$ and equal to any value greater than or equal to $\underline{p}_{\ell}$ and $r_{\ell}^{B}$ otherwise.

The first and third possibility of Step 4 can occur simultaneously, also in non-degenerate cases. In this case, the market for commodity $\ell$ is characterized by the absence of any supply and demand.

Step 5 of the proof of Theorem 6.1 defines prices of non-traded commodities in accordance with the requirements as coming out of Step 4. Step 6 defines rationing constraints on supply and demand in accordance with the requirements of Step 3 and Step 4. Step 7 verifies that the resulting prices, rationing schemes, and allocation satisfy Condition 1 of Definition 3.2. Step 8 does the same for Condition 2, Step 9 for Conditions 3, 4, 5, and 6, and Step 10 for Condition 7. This completes the proof of Theorem 6.1.

Theorem 5.2 asserts that a stable outcome exists. Using Theorem 6.1, we can then infer the existence of a Drèze equilibrium as a corollary.

Corollary 6.2. Consider an economy $\mathcal{E}=\left(T, \underline{p}, \bar{p},\left(u^{i}\right)_{i \in I}\right)$. A Drèze equilibrium exists.

The next result presents the converse of Theorem 6.1. If $\left(p^{*}, Q^{*}, R^{*}, x^{*}\right)$ is a Drèze equilibrium, then there is a stable outcome $A$ such that $\xi(A)=x^{*}$.

Theorem 6.3. Consider an economy $\mathcal{E}=\left(T, \underline{p}, \bar{p},\left(u^{i}\right)_{i \in I}\right)$. It holds that $X^{\mathrm{D}} \subset X^{\mathrm{so}}$.

Proof. See Appendix.

The proof of Theorem 6.3 consists of three easy steps. The first step takes some Drèze equilibrium and defines an associated outcome by specifying a matching between buyers and sellers that is compatible with the given Drèze equilibrium. The second step verifies individual rationality of the outcome and the third step absence of blocking by a two-player coalition.

Combining the results of Theorem 6.1 and Theorem 6.3 leads to the following corollary.

Corollary 6.4. Consider an economy $\mathcal{E}=\left(T, \underline{p}, \bar{p},\left(u^{i}\right)_{i \in I}\right)$. It holds that $X^{\mathrm{so}}=X^{\mathrm{D}}$. 
In the class of models under consideration, the set of stable outcomes coincides with the set of core outcomes. The relation between the core and competitive equilibrium is a classical problem in economics, dating back to Edgeworth (1881). In general, competitive equilibria belong to the core, but the core contains other allocations as well. In models of perfectly competitive economies, where agents are infinitely small, the core coincides with the set of competitive allocations. Debreu and Scarf (1963) obtain a core convergence result by showing that the set of core allocations shrinks to the competitive allocation when an economy is replicated. Aumann (1964) obtains a core equivalence result, showing that the core coincides with the set of competitive equilibrium allocations when there is a continuum of traders. This paper therefore delimits another class of economies where the core coincides with the set of equilibrium allocations. Moreover, agents need not be infinitely small. Where usually the equilibrium concepts are thought as more appealing in a set-up with many individuals that view themselves as an insignificant part of a large market and the cooperative concepts are thought to be more appropriate in situations with few players, both approaches coincide here irrespective of the number of agents.

\section{Conclusion}

This paper considers a discrete matching with contracts framework where contracts are subject to price controls. The framework is sufficiently general to admit many important models as special cases, like for instance the marriage problem of Gale and Shapley (1962), the assignment model without price controls of Shapley and Shubik (1971), the assignment model subject to price controls as in Talman and Yang (2008) or Andersson and Svensson (2014), and the quality competition model subject to price controls of Hatfield et al. (2012).

The paper presents an adjustment process that always terminates with a stable outcome for the discretized version of the model. For the marriage problem, the adjustment process coincides with the deferred acceptance algorithm, for the assignment model without price controls, the adjustment process coincides with the approximate auction mechanism as discussed in Section V of Demange et al. (1986), and in the case without ties, the adjustment process coincides with the generalized Gale-Shapley algorithm of Hatfield and Milgrom (2005). A limit argument demonstrates the existence of a stable outcome in a model without a smallest monetary unit.

The paper also develops an equilibrium concept for the framework under consideration. In the presence of price floors and price ceilings, rationing may be needed to clear the markets. In the equilibrium approach, rationing constraints cannot be arbitrary. Rationing on the demand side is only allowed in the presence of price ceilings and rationing on the demand side only in the presence of price floors. Rationing on supply and demand cannot occur simultaneously in a given market: markets are transparent. Buyers and sellers take prices and rationing constraints as given and maximize their utility given these constraints. At equilibrium, prices and rationing schemes are endogenously determined to equalize supply and demand of all commodities. The equilibrium concept is an extension of the concept of Drèze (1975) as developed for economies with divisible commodities.

The relationship between solution concepts coming from matching theory and those resulting from competitive analysis is an important topic of research. This paper contributes to this literature by showing that the set of stable allocations according to the matching approach is the same as the set of Drèze equilibrium allocations in the presence of price controls like price floors or price ceilings. Since price floors can be set equal to minus infinity and price ceilings to plus infinity, the case with absence of price floors and price ceilings is a special case. In this case, Drèze equilibria are equivalent to Walrasian equilibria and the result specializes to the equivalence be- 
tween Walrasian equilibria and stable outcomes. Since price floors and price ceilings can be both set equal to zero, the case without monetary transfers is a special case as well. As an example, the set of Drèze equilibria leads to a set of allocations that is equal to the one induced by stable outcomes in the marriage problem. Finally, there are many cases of interest in between unlimited monetary transfers and absence thereof, for instance, when studying the effects of minimum wages or rent controls.

Important generalizations of the current model concern the extension to the cases of manyto-one matching, many-to-many matching, and networks of agents who can trade contracts as in Ostrovsky (2008) and Hatfield et al. (2013). Such a generalization would require the adaptation of the concept of competitive equilibrium. Already in the case of many-to-one matching, it follows from examples in Kaneko (1982) that a product structure is not sufficient to guarantee that a single commodity trades against a single price. The appropriate competitive equilibrium concept would be one that involves personalized prices as in Hatfield et al. (2013). Existence would be another issue, since it follows from Hatfield and Kominers (2012) that without continuous transfers, in markets that lack a vertical structure, stable outcomes may not exist.

\section{Appendix A. Lemma A.1, Theorem A.2, Proof of Theorems 5.2, 6.1, and 6.3}

Lemma A.1. Consider an economy $\mathcal{E}=\left(T, \underline{p}, \bar{p},\left(u^{i}\right)_{i \in I}\right)$. Let $t=(b, s, \ell) \in T$ be such that there is $m \in\left[\underline{p}_{\ell}, \bar{p}_{\ell}\right] \cap \mathbb{R}$ such that $u^{b}(-m, \bar{e}(\ell)) \geq u^{b}\left(0,0^{L}\right)$ and $u^{s}(m,-e(\ell)) \geq u^{s}\left(0,0^{L}\right)$. Then there is $\underline{m}_{t}, \bar{m}_{t} \in \mathbb{R}$ with $\underline{p}_{\ell} \leq \underline{m}_{t} \leq \bar{m}_{t} \leq \bar{p}_{\ell}$ such that $(t, m) \in \bar{Y}$ is individually rational for both $b$ and $s$ if and only if $\bar{m} \in\left[\underline{m}_{t}, \bar{m}_{t}\right]$.

Proof. To define $\underline{m}_{t}$, we distinguish two cases. In the first case, $\underline{p}_{\ell}=-\infty$. Let $\underline{m}_{t} \in \mathbb{R}$ be the unique amount of money such that $u^{s}\left(\underline{m}_{t},-e(\ell)\right)=u^{s}\left(0,0^{L}\right)$. Since it has been assumed that for every $\ell \in L^{s}$ such that $\underline{p}_{\ell}=-\infty$, there is $m \in \mathbb{R}$ such that $u^{s}(m,-e(\ell)) \leq u^{s}\left(0,0^{L}\right)$ and there is $m \in\left[\underline{p}_{\ell}, \bar{p}_{\ell}\right] \cap \mathbb{R}$ such that $u^{s}(m,-e(\ell)) \geq u^{s}\left(0,0^{L}\right)$, and $u^{s}$ is strongly monotonic in $x_{0}$, it holds that $\underline{m}_{t}$ is uniquely determined. In the second case, it holds that $\underline{p}_{\ell} \neq-\infty$. Either $u^{s}\left(\underline{p}_{\ell},-e(\ell)\right) \geq u^{s}\left(0,0^{L}\right)$ and we define $\underline{m}_{t}=\underline{p}_{\ell}$, or $u^{s}\left(\underline{p}_{\ell},-e(\ell)\right)<u^{s}\left(0,0^{L}\right)$ and we define $\underline{m}_{t} \in \mathbb{R}$ as the unique amount of money such that $u^{s}\left(\underline{m}_{t},-e(\ell)\right)=u^{s}\left(0,0^{L}\right)$.

We define $\bar{m}_{t}$ in a similar way. If $\bar{p}_{\ell}=+\infty$, then let $\bar{m}_{t} \in \mathbb{R}$ be the unique amount of money such that $u^{b}\left(-\bar{m}_{t}, e(\ell)\right)=u^{b}\left(0,0^{L}\right)$. If $\bar{p}_{\ell} \neq+\infty$, then either $u^{b}\left(-\bar{p}_{\ell}, e(\ell)\right) \geq u^{b}\left(0,0^{L}\right)$ and we define $\bar{m}_{t}=\bar{p}_{\ell}$, or $u^{b}\left(-\bar{p}_{\ell}, e(\ell)\right)<u^{b}\left(0,0^{L}\right)$ and we define $\bar{m}_{t} \in \mathbb{R}$ as the unique amount of money such that $u^{b}\left(-\bar{m}_{t}, e(\ell)\right)=u^{b}\left(0,0^{L}\right)$.

It is easily verified that $\underline{p}_{\ell} \leq \underline{m}_{t} \leq \bar{m}_{t} \leq \bar{p}_{\ell}$.

It is immediate from the definitions of $\underline{m}_{t}$ and $\bar{m}_{t}$ that $(t, m) \in \bar{Y}$ is individually rational for both $b$ and $s$ if and only if $m \in\left[\underline{m}_{t}, \bar{m}_{t}\right]$.

Theorem A.2. The adjustment process of Definition 5.1 terminates in a finite number of steps with a set A of permanently accepted contracts. The set $A$ is a stable outcome for the set of feasible contracts $\bar{Y}^{\varepsilon}$.

Proof. Suppose the adjustment process does not terminate in a finite number of steps. Then, in Step 4 of each iteration, the set $Z \backslash A$ is non-empty and the cardinality of the set $Y$ in the next iteration diminishes by at least one. Since the initial set $Y$ as defined in Step 1 has finitely many elements, this leads to a contradiction. 
Let $A$ be the set of permanently accepted contracts. Since by Step 2 every buyer is involved in at most one contract in $A$, and by Step 3 every seller is involved in at most one contract in $A$, it follows that $A$ is an outcome.

For every $(m, t) \in \bar{Y}^{\varepsilon}$, the definitions of $\underline{m}_{t}$ and $\bar{m}_{t}$ guarantee individual rationality for both the buyer and the seller involved in trade $t$. It follows that Condition 1 of Definition 3.1 is satisfied.

Consider the finite sequence of sets of tentatively accepted contracts as generated in the various iterations of the adjustment process and the associated finite sequence of utilities of a seller $s$. The finite sequence of utilities is weakly increasing at each iteration. Indeed, the first, if any, tentatively accepted contract by seller $s$ gives utility at least equal to $U^{s}\left(0,0^{L}\right)$ since all contracts in $\bar{Y}^{\varepsilon}$ are individual rational for seller $s$. Next, a seller can keep a tentatively accepted contract forever, or rejects it in favor of a contract that gives at least the same utility.

Suppose there is $z=(b, s, \ell, m) \in \bar{Y}^{\varepsilon}$ such that $U^{b}(\{z\})>U^{b}(A)$ and $U^{s}(\{z\})>U^{s}(A)$. Then, at some iteration of the adjustment process, $z$ has been rejected in Step 3 by seller $s$. The tentatively accepted contract by seller $s$ in that iteration, say $y$, is such that $U^{s}(\{y\}) \geq U^{s}(\{z\})$. Since the utility of the tentatively accepted contract by seller $s$ is weakly increasing at each iteration, it holds that $U^{s}(A) \geq U^{s}(\{y\}) \geq U^{s}(\{z\})$, leading to a contradiction. Consequently, Condition 2 of Definition 3.1 is satisfied.

Theorem 5.2. Consider an economy $\mathcal{E}=\left(T, \underline{p}, \bar{p},\left(u^{i}\right)_{i \in I}\right)$. A stable outcome exists.

Proof. Consider the sequence $\left(\varepsilon_{n}\right)_{n \in \mathbb{N}}$ with $\varepsilon_{n}=2^{-n}$ and let $A_{n}$ be the outcome that is generated by the adjustment process of Definition 5.1 for the set of feasible contracts $\bar{Y}^{\varepsilon_{n}}$. By listing the, potentially empty, contracts of the buyers, we can represent every $A_{n}$ as an element of the set

$$
(S \times L \times[\underline{m}, \bar{m}] \cup\{\emptyset\})^{B},
$$

where $\underline{m}$ is a finite lower bound for $\left\{\underline{m}_{t} \mid t \in T\right\}$ and $\bar{m}$ is a finite upper bound for $\left\{\bar{m}_{t} \mid t \in T\right\}$. We can identify the elements of $S, L$, and $\{\emptyset\}$ with distinct points in Euclidean space. Then the set in (A.1) is compact under the induced topology. The sequence $A_{n}$ therefore has a convergent subsequence, denoted by $\left(A_{n_{k}}\right)_{k \in \mathbb{N}}$, with limit, say, $A$.

The remainder of the proof verifies that $A$ is a stable outcome.

It clearly holds that $A \subset \bar{Y}$ is an outcome and that every contract $(t, m) \in A$ satisfies $\underline{m}_{t} \leq m \leq$ $\bar{m}_{t}$. Condition 1 of Definition 3.1 corresponding to individual rationality is therefore satisfied by $A$.

Suppose that there is $z=(b, s, \ell, m) \in \bar{Y}$ such that $U^{b}(\{z\})>U^{b}(A)$ and $U^{s}(\{z\})>U^{s}(A)$. Since $A$ satisfies individual rationality, it follows that $\underline{m}_{b, s, \ell} \leq m \leq \bar{m}_{b, s, \ell}$. Then there is $n^{\prime} \in \mathbb{N}$ and $y=(b, s, \ell, \tilde{m}) \in \bar{Y}^{\varepsilon_{n^{\prime}}}$ such that $U^{b}(\{y\})>U^{b}(A)$ and $U^{s}(\{y\})>U^{s}(A)$. Observe that, for every $n \geq n^{\prime}, y \in \bar{Y}^{\varepsilon_{n}}$.

Let $k^{\prime}$ be such that, for every $k \geq k^{\prime}, U^{b}(\{y\})>U^{b}\left(A_{n_{k}}\right)$ and $U^{s}(\{y\})>U^{s}\left(A_{n_{k}}\right)$. Let $k^{*}$ be such that $n_{k^{*}} \geq \max \left\{n^{\prime}, n_{k^{\prime}}\right\}$. It follows that $b$ and $s$ can use $y \in \bar{Y}^{\varepsilon_{n_{k^{*}}}}$ to block outcome $A_{n_{k^{*}}}$, thereby violating that $A_{n_{k^{*}}}$ is stable for the set of feasible contracts $\bar{Y}^{\varepsilon_{n} k^{*}}$. Consequently, $A$ satisfies Condition 2 of Definition 3.1.

Theorem 6.1. Consider an economy $\mathcal{E}=\left(T, \underline{p}, \bar{p},\left(u^{i}\right)_{i \in I}\right)$. It holds that $X^{\mathrm{so}} \subset X^{\mathrm{D}}$.

Proof. Consider some $x^{*} \in X^{\text {so }}$ and let $A$ be a stable outcome such that $\xi(A)=x^{*}$. We prove the result in ten steps. 
Step 1 . A commodity trades at a single price: For every $y, y^{\prime} \in A$ such that $\lambda(y)=\lambda\left(y^{\prime}\right)$ it holds that $\mu(y)=\mu\left(y^{\prime}\right)$.

Suppose $\mu(y) \neq \mu\left(y^{\prime}\right)$ and without loss of generality assume $\mu(y)<\mu\left(y^{\prime}\right)$. Consider the contract

$$
z=\left(b, s, \lambda(y), \frac{1}{2} \mu(y)+\frac{1}{2} \mu\left(y^{\prime}\right)\right),
$$

where $b=\beta\left(y^{\prime}\right)$ and $s=\sigma(y)$. Since

$$
\underline{p}_{\lambda(y)} \leq \mu(y)<\frac{1}{2} \mu(y)+\frac{1}{2} \mu\left(y^{\prime}\right)<\mu\left(y^{\prime}\right) \leq \bar{p}_{\lambda(y)},
$$

and the set of traders in commodity $\ell$ has a product structure, it holds that $z \in \bar{Y}$. The utilities corresponding to $z$ are equal to

$$
\begin{aligned}
& U^{b}(\{z\})=u^{b}\left(-\frac{1}{2} \mu(y)-\frac{1}{2} \mu\left(y^{\prime}\right), e(\lambda(y))\right)>u^{b}\left(-\mu\left(y^{\prime}\right), e\left(\lambda\left(y^{\prime}\right)\right)\right)=U^{b}(A), \\
& U^{s}(\{z\})=u^{s}\left(\frac{1}{2} \mu(y)+\frac{1}{2} \mu\left(y^{\prime}\right),-e(\lambda(y))\right)>u^{s}(\mu(y),-e(\lambda(y)))=U^{s}(A),
\end{aligned}
$$

so Condition 2 of Definition 3.1 is violated and the outcome $A$ is not stable, a contradiction. Consequently, for every $y, y^{\prime} \in A$ such that $\lambda(y)=\lambda\left(y^{\prime}\right)$ it holds that $\mu(y)=\mu\left(y^{\prime}\right)$.

Step 2. Definition of prices for traded commodities and reservation values for all commodities.

For every $\ell \in \lambda(A)$, we denote the price at which $\ell$ is traded by $p_{\ell}^{*}$, so for every $y \in A$ such that $\lambda(y)=\ell$ it holds that $\mu(y)=p_{\ell}^{*}$.

Consider some $\ell \in L$ and some $b \in \beta\left(\bar{Y}_{\ell}\right)$. If, for every $m \in \mathbb{R}, u^{b}(-m, e(\ell))>u^{b}\left(x^{* b}\right)$, then we define $r_{\ell}^{b}=+\infty$ and $u^{b}(-\infty, e(\ell))=\lim _{m \rightarrow+\infty} u^{b}(-m, e(\ell))$. Notice that by the assumptions on $u^{b}$, it holds that $\bar{p}_{\ell}<+\infty$ in this case. It also holds that $u^{b}(-\infty, e(\ell)) \geq u^{b}\left(x^{* b}\right)$. If, for every $m \in \mathbb{R}, u^{b}(-m, e(\ell))<u^{b}\left(x^{* b}\right)$, then we define $r_{\ell}^{b}=-\infty$ and $u^{b}(+\infty, e(\ell))=$ $\lim _{m \rightarrow-\infty} u^{b}(-m, e(\ell))$. It holds that $u^{b}(+\infty, e(\ell)) \leq u^{b}\left(x^{* b}\right)$. Otherwise, let $r_{\ell}^{b} \in \mathbb{R}$ be such that $u^{b}\left(-r_{\ell}^{b}, e(\ell)\right)=u^{b}\left(x^{* b}\right)$. We define $r_{\ell}^{B}=\max _{b \in \beta\left(\bar{Y}_{\ell}\right)} r_{\ell}^{b}$.

Consider some $\ell \in L$ and some $s \in \sigma\left(\bar{Y}_{\ell}\right)$. If, for every $m \in \mathbb{R}, u^{s}(m,-e(\ell))>u^{s}\left(x^{* s}\right)$, then we define $r_{\ell}^{s}=-\infty$ and $u^{s}(-\infty,-e(\ell))=\lim _{m \rightarrow-\infty} u^{s}(m,-e(\ell))$. Notice that by the assumptions on $u^{s}$, it holds that $\underline{p}_{\ell}>-\infty$ in this case. It also holds that $u^{s}(-\infty,-e(\ell)) \geq u^{s}\left(x^{* s}\right)$. If, for every $m \in \mathbb{R}, u^{s}(m,-e(\ell))<u^{s}\left(x^{* s}\right)$, then we define $r_{\ell}^{s}=+\infty$ and $u^{s}(+\infty,-e(\ell))=$ $\lim _{m \rightarrow+\infty} u^{s}(m,-e(\ell))$. It holds that $u^{s}(+\infty,-e(\ell)) \leq u^{s}\left(x^{* s}\right)$. Otherwise, let $r_{\ell}^{s} \in \mathbb{R}$ be such that $u^{s}\left(r_{\ell}^{s},-e(\ell)\right)=u^{s}\left(x^{* s}\right)$. We define $r_{\ell}^{S}=\min _{s \in \sigma\left(\bar{Y}_{\ell}\right)} r_{\ell}^{s}$.

It holds by definition that $r_{\ell}^{b}=p_{\ell}^{*}=r_{\ell}^{s}$ for every $(b, s, \ell, m) \in A$.

Step 3. For every $\ell \in \lambda(A)$ at least one of the following three cases holds:

1. $p_{\ell}^{*}=\underline{p}_{\ell}$ and $r_{\ell}^{S} \leq \underline{p}_{\ell}=r_{\ell}^{B}$,

2. $\underline{p}_{\ell}<p_{\ell}^{*}<\bar{p}_{\ell}$ and $r_{\ell}^{S}=p_{\ell}^{*}=r_{\ell}^{B}$,

3. $p_{\ell}^{*}=\bar{p}_{\ell}$ and $r_{\ell}^{S}=\bar{p}_{\ell} \leq r_{\ell}^{B}$.

It follows from the definitions in Step 2 that, for every $(b, s, \ell, m) \in A, r_{\ell}^{b}=p_{\ell}^{*}=r_{\ell}^{s}$, so it follows that $r_{\ell}^{S} \leq p_{\ell}^{*} \leq r_{\ell}^{B}$.

Consider some $\ell \in L$. It clearly holds that a. $p_{\ell}^{*}=\underline{p}_{\ell}$, or b. $\underline{p}_{\ell}<p_{\ell}^{*}<\bar{p}_{\ell}$, or c. $p_{\ell}^{*}=\bar{p}_{\ell}$. Each of the Cases a, b, and c is considered in turn and shown to lead to at least one of the Cases 1,2 , and 3 of Step 3.

Case a. $p_{\ell}^{*}=\underline{p}_{\ell}$. 
If $r_{\ell}^{B}=\underline{p}_{\ell}$, then Case 1 of Step 3 holds and we are done, so assume $r_{\ell}^{B}>\underline{p}_{\ell}$. We argue that $r_{\ell}^{S}=\underline{p}_{\ell}$. Suppose not, then $r_{\ell}^{S} \leq p_{\ell}^{*}=\underline{p}_{\ell}$ implies $r_{\ell}^{S}<\underline{p}_{\ell}$. Let $b \in \beta\left(\bar{Y}_{\ell}\right)$ be such that $r_{\ell}^{b}=r_{\ell}^{B}$, let $s \in \sigma\left(\bar{Y}_{\ell}\right)$ be such that $r_{\ell}^{s}=r_{\ell}^{S}$, and consider the contract $z=\left(b, s, \ell, \underline{p}_{\ell}\right)$. Since the set of traders in commodity $\ell$ has a product structure, it holds that $z \in \bar{Y}$. The utilities corresponding to $z$ are equal to

$$
\begin{aligned}
& U^{b}(\{z\})=u^{b}\left(-\underline{p}_{\ell}, e(\ell)\right)>u^{b}\left(-r_{\ell}^{b}, e(\ell)\right) \geq u^{b}\left(x^{* b}\right), \\
& U^{s}(\{z\})=u^{s}\left(\underline{p}_{\ell},-e(\ell)\right)>u^{s}\left(r_{\ell}^{s},-e(\ell)\right) \geq u^{s}\left(x^{* s}\right),
\end{aligned}
$$

so Condition 2 of Definition 3.1 is violated and the outcome $A$ is not stable, a contradiction. Consequently, it holds that $r_{\ell}^{S}=\underline{p}_{\ell}$.

We argue next that $\underline{p}_{\ell}=\bar{p}_{\ell}$. Suppose not, then it holds that $\underline{p}_{\ell}<\bar{p}_{\ell}$. Let $b \in \beta\left(\bar{Y}_{\ell}\right)$ be such that $r_{\ell}^{b}=r_{\ell}^{B}$, let $s \in \sigma\left(\bar{Y}_{\ell}\right)$ be such that $r_{\ell}^{s}=r_{\ell}^{S}$, and let $m \in \mathbb{R}$ be such that $\underline{p}_{\ell}<m<$ $\min \left\{\bar{p}_{\ell}, r_{\ell}^{B}\right\}$. The contract $z=(b, s, \ell, m)$ belongs to $\bar{Y}$ since the set of traders in commodity $\ell$ has a product structure and $\underline{p}_{\ell}<m<\bar{p}_{\ell}$. The utilities corresponding to $z$ are equal to

$$
\begin{aligned}
& U^{b}(\{z\})=u^{b}(-m, e(\ell))>u^{b}\left(-r_{\ell}^{b}, e(\ell)\right) \geq u^{b}\left(x^{* b}\right), \\
& U^{s}(\{z\})=u^{s}(m,-e(\ell))>u^{s}\left(\underline{p}_{\ell},-e(\ell)\right)=u^{s}\left(r_{\ell}^{s},-e(\ell)\right) \geq u^{s}\left(x^{* s}\right) .
\end{aligned}
$$

Condition 2 of Definition 3.1 is violated and the outcome $A$ is not stable, a contradiction. Consequently, it holds that $\underline{p}_{\ell}=\bar{p}_{\ell}$. Now Case 3 of Step 3 holds and we are done.

Case b. $\underline{p}_{\ell}<p_{\ell}^{*}<\bar{p}_{\ell}$.

Suppose that Case 2 of Step 3 does not hold. We end up in Subcase (b.i). $r_{\ell}^{S}<p_{\ell}^{*} \leq r_{\ell}^{B}$ or Subcase (b.ii). $r_{\ell}^{S} \leq p_{\ell}^{*}<r_{\ell}^{B}$.

In Subcase (b.i), let $b \in \beta\left(\bar{Y}_{\ell}\right)$ be such that $r_{\ell}^{b}=r_{\ell}^{B}$, let $s \in \sigma\left(\bar{Y}_{\ell}\right)$ be such that $r_{\ell}^{s}=r_{\ell}^{S}$, and let $m \in \mathbb{R}$ be such that $\max \left\{\underline{p}_{\ell}, r_{\ell}^{S}\right\}<m<p_{\ell}^{*}$. The contract $z=(b, s, \ell, m)$ belongs to $\bar{Y}$ since the set of traders in commodity $\ell$ has a product structure and $\underline{p}_{\ell}<m<\bar{p}_{\ell}$. The utilities corresponding to $z$ are equal to

$$
\begin{aligned}
& U^{b}(\{z\})=u^{b}(-m, e(\ell))>u^{b}\left(-p_{\ell}^{*}, e(\ell)\right) \geq u^{b}\left(-r_{\ell}^{b}, e(\ell)\right) \geq u^{b}\left(x^{* b}\right), \\
& U^{s}(\{z\})=u^{s}(m,-e(\ell))>u^{s}\left(r_{\ell}^{s},-e(\ell)\right) \geq u^{s}\left(x^{* s}\right),
\end{aligned}
$$

so Condition 2 of Definition 3.1 is violated and the outcome $A$ is not stable, a contradiction.

In Subcase (b.ii), let $b \in \beta\left(\bar{Y}_{\ell}\right)$ be such that $r_{\ell}^{b}=r_{\ell}^{B}$, let $s \in \sigma\left(\bar{Y}_{\ell}\right)$ be such that $r_{\ell}^{s}=r_{\ell}^{S}$, and let $m \in \mathbb{R}$ be such that $p_{\ell}^{*}<m<\min \left\{\bar{p}_{\ell}, r_{\ell}^{B}\right\}$. The contract $z=(b, s, \ell, m)$ belongs to $\bar{Y}$ since the set of traders in commodity $\ell$ has a product structure and $\underline{p}_{\ell}<m<\bar{p}_{\ell}$. The utilities corresponding to $z$ are equal to

$$
\begin{aligned}
& U^{b}(\{z\})=u^{b}(-m, e(\ell))>u^{b}\left(-r_{\ell}^{b}, e(\ell)\right) \geq u^{b}\left(x^{* b}\right), \\
& U^{s}(\{z\})=u^{s}(m,-e(\ell))>u^{s}\left(p_{\ell}^{*},-e(\ell)\right) \geq u^{s}\left(r_{\ell}^{s},-e(\ell)\right) \geq u^{s}\left(x^{* s}\right),
\end{aligned}
$$

so Condition 2 of Definition 3.1 is violated and the outcome $A$ is not stable, a contradiction.

Since both Subcases (b.i) and (b.ii) lead to a contradiction, our supposition is false, so Case 2 of Step 3 holds and we are done.

Case c. $p_{\ell}^{*}=\bar{p}_{\ell}$.

By an argument completely symmetric to the one of Case a, Case 1 or Case 3 of Step 3 can be shown to hold. 
Step 4. For every $\ell \in L \backslash \lambda(A)$, at least one of the following three cases holds:

1. $r_{\ell}^{B} \leq \underline{p}_{\ell}$

2. $\underline{p}_{\ell}<r_{\ell}^{B} \leq r_{\ell}^{S}<\bar{p}_{\ell}$,

3. $r_{\ell}^{S} \geq \bar{p}_{\ell}$.

Suppose not. Then there is $\ell \in L \backslash \lambda(A)$ such that

$$
r_{\ell}^{B}>\underline{p}_{\ell}, r_{\ell}^{S}<\bar{p}_{\ell}, \text { and } r_{\ell}^{B}>r_{\ell}^{S} .
$$

Take $b \in \beta\left(\bar{Y}_{\ell}\right)$ with $r_{\ell}^{b}=r_{\ell}^{B}$ and $s \in \sigma\left(\bar{Y}_{\ell}\right)$ with $r_{\ell}^{s}=r_{\ell}^{S}$. Since $r_{\ell}^{B} \neq-\infty$ and $r_{\ell}^{S} \neq+\infty$, it holds that $u^{b}\left(-r_{\ell}^{b}, e(\ell)\right) \geq u^{b}\left(x^{* b}\right)$ and $u^{s}\left(r_{\ell}^{s},-e(\ell)\right) \geq u^{s}\left(x^{* s}\right)$. Let $m \in \mathbb{R}$ be such that $\underline{p}_{\ell} \leq$ $m \leq \bar{p}_{\ell}$ and $r_{\ell}^{S}<m<r_{\ell}^{B}$. Since $\underline{p}_{\ell} \leq m \leq \bar{p}_{\ell}$ and the set of traders in commodity $\ell$ has a product structure, it holds that $z=(b, s, m, \ell) \in \bar{Y}$. The utilities corresponding to $z$ are equal to

$$
\begin{aligned}
& U^{b}(\{z\})=u^{b}(-m, e(\ell))>u^{b}\left(-r_{\ell}^{b}, e(\ell)\right) \geq u^{b}\left(x^{* b}\right), \\
& U^{s}(\{z\})=u^{s}(m,-e(\ell))>u^{s}\left(r_{\ell}^{s},-e(\ell)\right) \geq u^{s}\left(x^{* s}\right),
\end{aligned}
$$

so Condition 2 of Definition 3.1 is violated and the outcome $A$ is not stable, a contradiction. The result as stated in Step 4 follows.

Step 5. Definition of prices for non-traded commodities.

Choose any $\ell \in L \backslash \lambda(A)$. Using Step 4, exactly one of the following three cases holds.

Case 1. $r_{\ell}^{B} \leq \underline{p}_{\ell}$. If $\underline{p}_{\ell}>-\infty$, then define $p_{\ell}^{*}=\underline{p}_{\ell}$. Consider the case $\underline{p}_{\ell}=-\infty$. Since $\underline{p}_{\ell}=-\infty$, it holds that $r_{\ell}^{S}>-\infty$. Define $p_{\ell}^{*}=\min \left\{\bar{p}_{\ell}, r_{\ell}^{S}, 0\right\}$.

Case 2. $\underline{p}_{\ell}<r_{\ell}^{B} \leq r_{\ell}^{S}<\bar{p}_{\ell}$. Define $p_{\ell}^{*}=\left(r_{\ell}^{B}+r_{\ell}^{S}\right) / 2$.

Case 3. $\underline{p}_{\ell}<r_{\ell}^{B}$ and $\bar{p}_{\ell} \leq r_{\ell}^{S}$. If $\bar{p}_{\ell}<+\infty$, then define $p_{\ell}^{*}=\bar{p}_{\ell}$. If $\bar{p}_{\ell}=+\infty$, then define $p_{\ell}^{*}=r_{\ell}^{B}$.

Step 6. Definition of rationing constraints.

For every buyer, we introduce rationing constraints for those commodities that he does not trade but would like to trade and which have a price equal to the price ceiling,

$$
Q^{*}=\left\{(b, \ell) \in B \times L \mid \ell \in L^{b} \backslash \lambda\left(A^{b}\right) \text { and } r_{\ell}^{b}>p_{\ell}^{*}=\bar{p}_{\ell}\right\} .
$$

For every seller, we introduce rationing constraints for those commodities that he does not trade but would like to trade and which have a price equal to the price floor,

$$
R^{*}=\left\{(s, \ell) \in S \times L \mid \ell \in L^{s} \backslash \lambda\left(A^{s}\right) \text { and } r_{\ell}^{s}<p_{\ell}^{*}=\underline{p}_{\ell}\right\} .
$$

Step 7. ( $\left.p^{*}, Q^{*}, R^{*}, x^{*}\right)$ satisfies Condition 1 of Definition 3.2.

Consider some $b \in B$. Suppose $x^{* b} \notin \delta^{b}\left(p^{*}, Q^{* b}\right)$. Take some $x^{b} \in \delta^{b}\left(p^{*}, Q^{* b}\right)$. It follows that $x^{b} \neq 0$ since otherwise $A^{b} \notin C^{b}(A)$, thereby violating Condition 1 of Definition 3.1. Let $\ell \in L^{b}$ be such that $x_{\ell}^{b}=1$. It follows that $\ell \notin Q^{* b}$. By (A.2) it must be that $\ell \in \lambda\left(A^{b}\right)$ or $r_{\ell}^{b} \leq p_{\ell}^{*}$ or $p_{\ell}^{*}<\bar{p}_{\ell}$. The first two cases imply $u^{b}\left(x^{b}\right)=u^{b}\left(x^{* b}\right)$ and $u^{b}\left(x^{b}\right) \leq u^{b}\left(x^{* b}\right)$, respectively, leading to a contradiction. For the last case we distinguish two subcases: (i) $\ell \in L^{b} \backslash \lambda(A)$ and (ii) $\ell \in \lambda(A)$.

For subcase (i), using $p_{\ell}^{*}<\bar{p}_{\ell}$, it follows from the definition in Step 5 that $r_{\ell}^{B} \leq p_{\ell}^{*}$. At the same time, $u^{b}\left(x^{b}\right)>u^{b}\left(x^{* b}\right)$ implies $p_{\ell}^{*}<r_{\ell}^{b} \leq r_{\ell}^{B}$, so we have obtained a contradiction. 
For subcase (ii), since $u^{b}\left(x^{b}\right)>u^{b}\left(x^{* b}\right)$, it holds that $p_{\ell}^{*}<r_{\ell}^{b} \leq r_{\ell}^{B}$, so Cases 1 and 2 of Step 3 do not hold. Since $p_{\ell}^{*}<\bar{p}_{\ell}$, Case 3 of Step 3 is violated as well, so we have obtained a contradiction. Consequently, it holds that $x^{* b} \in \delta^{b}\left(p^{*}, Q^{* b}\right)$.

Step 8. $\left(p^{*}, Q^{*}, R^{*}, x^{*}\right)$ satisfies Condition 2 of Definition 3.2.

Consider some $s \in S$. Suppose $x^{* s} \notin \delta^{s}\left(p^{*}, R^{* s}\right)$. Take some $x^{s} \in \delta^{s}\left(p^{*}, R^{* s}\right)$. It follows that $x^{s} \neq 0$ since otherwise $A^{s} \notin C^{s}(A)$, thereby violating Condition 1 of Definition 3.1. Let $\ell \in L^{s}$ be such that $x_{\ell}^{s}=-1$. It follows that $\ell \notin R^{* s}$. By (A.3) it must be that $\ell \in \lambda\left(A^{s}\right)$ or $r_{\ell}^{s} \geq p_{\ell}^{*}$ or $p_{\ell}^{*}>\underline{p}_{\ell}$. The first two cases imply $u^{s}\left(x^{s}\right)=u^{s}\left(x^{* s}\right)$ and $u^{s}\left(x^{s}\right) \leq u^{s}\left(x^{* s}\right)$, respectively, leading to a contradiction. For the last case we distinguish two subcases: (i) $\ell \in L^{s} \backslash \lambda(A)$ and (ii) $\ell \in \lambda(A)$.

For subcase (i), using $p_{\ell}^{*}>\underline{p}_{\ell}$, it follows from the definition in Step 5 that $r_{\ell}^{S} \geq p_{\ell}^{*}$. At the same time, $u^{s}\left(x^{s}\right)>u^{s}\left(x^{* s}\right)$ implies $p_{\ell}^{*}>r_{\ell}^{s} \geq r_{\ell}^{S}$, so we have obtained a contradiction.

For subcase (ii), since $u^{s}\left(x^{s}\right)>u^{s}\left(x^{* s}\right)$, it holds that $p_{\ell}^{*}>r_{\ell}^{s} \geq r_{\ell}^{S}$, so Cases 2 and 3 of Step 3 do not hold. Since $p_{\ell}^{*}>p_{\ell}$, Case 1 of Step 3 is violated as well, so we have obtained a contradiction. Consequently, it holds that $x^{* s} \in \delta^{s}\left(p^{*}, R^{* s}\right)$.

Step 9. ( $\left.p^{*}, Q^{*}, R^{*}, x^{*}\right)$ satisfies Conditions 3, 4, 5, and 6 of Definition 3.2.

It follows from (3.1) that

$$
\sum_{i \in I} x^{* i}=\sum_{i \in I} \xi^{i}(A)=0,
$$

and therefore Condition 3 of Definition 3.2 is satisfied.

The definition of prices for traded commodities in Step 2 implies that for every $\ell \in \lambda(A)$, $\underline{p}_{\ell} \leq p_{\ell}^{*} \leq \bar{p}_{\ell}$. The definition of prices for non-traded commodities in Step 5 implies that for every $\ell \in L \backslash \lambda(A), \underline{p}_{\ell} \leq p_{\ell}^{*} \leq \bar{p}_{\ell}$. Therefore, Condition 4 of Definition 3.2 is satisfied.

It follows from (A.2) that, for every $\ell \in L$, if $p_{\ell}^{*}<\bar{p}_{\ell}$, then $Q_{\ell}^{*}=\emptyset$, and therefore Condition 5 of Definition 3.2 is satisfied. It follows from (A.3) that, for every $\ell \in L$, if $p_{\ell}^{*}>\underline{p}_{\ell}$, then $R_{\ell}^{*}=\emptyset$, and therefore Condition 6 of Definition 3.2 is satisfied.

Step 10. $\left(p^{*}, Q^{*}, R^{*}, x^{*}\right)$ satisfies Condition 7 of Definition 3.2.

Suppose there is $\ell \in L$ such that $Q_{\ell}^{*} \neq \emptyset$ and $R_{\ell}^{*} \neq \emptyset$. From (A.2) and (A.3) it follows that there is $b \in B$ and $s \in S$ such that $\underline{p}_{\ell}=p_{\ell}^{*}=\bar{p}_{\ell}$ and $r_{\ell}^{s}<p_{\ell}^{*}<r_{\ell}^{b}$, so in particular $r_{\ell}^{S}<p_{\ell}^{*}<r_{\ell}^{B}$. If $\ell \in \lambda(A)$, then none of the cases in Step 3 holds, a contradiction. If $\ell \in L \backslash \lambda(A)$, then none of the cases in Step 4 holds, a contradiction. Consequently, for every $\ell \in L, Q_{\ell}^{*}=\emptyset$ or $R_{\ell}^{*}=\emptyset$, and Condition 7 of Definition 3.2 is satisfied.

We conclude that $\left(p^{*}, Q^{*}, R^{*}, x^{*}\right)$ is a Drèze equilibrium and therefore $x^{*} \in X^{\mathrm{D}}$.

Theorem 6.3. Consider an economy $\mathcal{E}=\left(T, \underline{p}, \bar{p},\left(u^{i}\right)_{i \in I}\right)$. It holds that $X^{\mathrm{D}} \subset X^{\mathrm{so}}$.

Proof. Step 1. Definition of a stable outcome.

By Corollary 6.2, the set $X^{D}$ is non-empty. Take some $x^{*} \in X^{\mathrm{D}}$ and choose $\left(p^{*}, Q^{*}, R^{*}\right) \in$ $\left(\{1\} \times \mathbb{R}^{L}\right) \times \mathcal{Q} \times \mathcal{R}$ such that $\left(p^{*}, Q^{*}, R^{*}, x^{*}\right)$ is a Drèze equilibrium. For every $\ell \in L$ with $x_{\ell}^{*} \neq 0$, Condition 3 of Definition 3.2 implies that there are exactly as many buyers as sellers. Match every buyer $b \in B$ with $x_{\ell}^{* b}=1$ to a seller $s(b) \in S$ with $x_{\ell}^{* s(b)}=-1$, so in particular $b \neq$ $b^{\prime}$ implies $s(b) \neq s\left(b^{\prime}\right)$, and define a contract $y^{b}=\left(b, s(b), \ell, p_{\ell}^{*}\right)$. We define $A$ as the set of all such contracts, $A=\cup_{\left\{b \in B \mid x^{* b} \neq\left(0,0^{L}\right)\right\}}\left\{y^{b}\right\}$. It clearly holds that, for every $i \in I, U^{i}(A)=u^{i}\left(x^{* i}\right)$, and $\xi(A)=x^{*}$.

Step 2. For every $i \in I, A^{i} \in C^{i}(A)$. 
Suppose for some $b \in B, A^{b} \notin C^{b}(A)$, so $u^{b}\left(0,0^{L}\right)>u^{b}\left(x^{* b}\right)$, a contradiction to $x^{* b} \in$ $\delta^{b}\left(p^{*}, Q^{* b}\right)$. Consequently, it holds for every $b \in B$ that $A^{b} \in C^{b}(A)$.

Suppose for some $s \in S, A^{s} \notin C^{s}(A)$, so $u^{s}\left(0,0^{L}\right)>u^{s}\left(x^{* s}\right)$, a contradiction to $x^{* s} \in$ $\delta^{s}\left(p^{*}, R^{* s}\right)$. Consequently, it holds for every $s \in S$ that $A^{s} \in C^{s}(A)$.

It follows that Condition 1 of Definition 3.1 is satisfied.

Step 3. For every $z \in \bar{Y}$ it holds that $U^{b}(\{z\}) \leq U^{b}(A)$ or $U^{s}(\{z\}) \leq U^{s}(A)$.

Suppose there is $z=(b, s, \ell, m) \in \bar{Y}$ such that $U^{b}(\{z\})>U^{b}(A)=u^{b}\left(x^{* b}\right)$ and $U^{s}(\{z\})>$ $U^{s}(A)=u^{s}\left(x^{* s}\right)$. By Condition 7 of Definition 3.2, it holds that $Q_{\ell}^{*}=\emptyset$ or $R_{\ell}^{*}=\emptyset$.

Consider the case with $Q_{\ell}^{*}=\emptyset$ first. Since $x^{* b} \in \delta^{b}\left(p^{*}, Q^{* b}\right)$ and $U^{b}(\{z\})>u^{b}\left(x^{* b}\right)$, it holds that $(-m, e(\ell)) \notin \gamma^{b}\left(p^{*}, Q^{* b}\right)$. Since there is no demand rationing of commodity $\ell$, it now follows that $m<p_{\ell}^{*}$. Since $z \in \bar{Y}$, this implies in turn that $\underline{p}_{\ell}<p_{\ell}^{*}$, so, according to Condition 6 of Definition 3.2, $R_{\ell}^{*}=\emptyset$. Since there is no supply rationing of commodity $\ell, U^{s}(\{z\})>u^{s}\left(x^{* s}\right)$ implies that $m>p_{\ell}^{*}$, and we have obtained a contradiction.

Consider next the case with $R_{\ell}^{*}=\emptyset$. Since $x^{* s} \in \delta^{s}\left(p^{*}, R^{* s}\right)$ and $U^{s}(\{z\})>u^{s}\left(x^{* s}\right)$, it holds that $(m,-e(\ell)) \notin \gamma^{s}\left(p^{*}, R^{* s}\right)$. Since there is no supply rationing of commodity $\ell$, it now follows that $m>p_{\ell}^{*}$. Since $z \in \bar{Y}$, this implies in turn that $\bar{p}_{\ell}>p_{\ell}^{*}$, so, according to Condition 5 of Definition $3.2, Q_{\ell}^{*}=\emptyset$. Since there is no demand rationing of commodity $\ell, U^{b}(\{z\})>u^{b}\left(x^{* b}\right)$ implies that $m<p_{\ell}^{*}$, and again we have obtained a contradiction.

It follows that Condition 2 of Definition 3.1 is satisfied.

The outcome $A$ is stable and therefore $\xi(A)=x^{*} \in X^{\text {so }}$.

\section{References}

Agell, J., Lommerud, K.E., 1997. Minimum wages and the incentives for skill formation. J. Public Econ. 64, 25-40. Andersson, T., Svensson, L.G., 2014. Non-manipulable house allocation with rent control. Econometrica 82, 507-539. Aumann, R.J., 1964. Markets with a continuum of traders. Econometrica 32, 39-50.

Bénassy, J.-P., 1975. Neo-keynesian disequilibrium theory in a monetary economy. Rev. Econ. Stud. 42, 503-523.

Crawford, V.P., Knoer, E.M., 1981. Job matching with heterogeneous firms and workers. Econometrica 49, 437-450.

Debreu, G., Scarf, H., 1963. A limit theorem on the core of an economy. Int. Econ. Rev. 4, 235-246.

Demange, G., Gale, D., Sotomayor, M., 1986. Multi-item auctions. J. Polit. Econ. 94, 863-872.

Drèze, J.H., 1975. Existence of an exchange equilibrium under price rigidities. Int. Econ. Rev. 16, 301-320.

Edgeworth, F.Y., 1881. Mathematical Psychics. Kegan Paul, London.

Feldstein, M., 1973. The economics of the new unemployment. Public Interest 33, 3-42.

Gale, D., Shapley, L.S., 1962. College admissions and the stability of marriage. Am. Math. Mon. 69, 9-15.

Gul, F., Stacchetti, E., 1999. Walrasian equilibrium with Gross substitutes. J. Econ. Theory 87, 95-124.

Hatfield, J.W., Kominers, S.D., 2012. Matching in networks with bilateral contracts. Am. Econ. J. Microecon. 4, 176-208.

Hatfield, J.W., Kominers, S.D., Nichifor, A., Ostrovsky, M., Westkamp, A., 2013. Stability and competitive equilibrium in trading networks. J. Polit. Econ. 121, 966-1005.

Hatfield, J.W., Milgrom, P., 2005. Matching with contracts. Am. Econ. Rev. 95, 915-935.

Hatfield, J.W., Plott, C.R., Tanaka, T., 2012. Understanding price controls and nonprice competition with matching theory. Am. Econ. Rev. Pap. Proc. 102, 371-375.

Hatfield, J.W., Plott, C.R., Tanaka, T., 2016. Price controls, non-price quality competition, and the nonexistence of competitive equilibrium. Games Econ. Behav. 99, 134-163.

Herings, P.J.J., 1996. Static and dynamic aspects of general disequilibrium theory. In: Theory and Decision Library, Series C: Game Theory, Mathematical Programming and Operations Research. Kluwer Academic Publishers, Norwell, Massachusetts.

Herings, P.J.J., Konovalov, A., 2009. Constrained suboptimality when prices are non-competitive. J. Math. Econ. 45, 43-58.

Jaffe, S., Kominers, S.D., 2014. Taxation in Matching Markets. Working Paper. pp. 1-48.

Kaneko, M., 1982. The central assignment game and the assignment markets. J. Math. Econ. 10, $205-232$.

Kelso, A.S. Jr., Crawford, V.P., 1982. Job matching, coalition formation, and gross substitutes. Econometrica 50, 1483-1504. 
Leffler, K.B., 1982. Ambiguous changes in product quality. Am. Econ. Rev. 72, 956-967.

Levy, S., 1991. The short-term macroeconomic effects of price controls. J. Policy Model. 13, 157-184.

Nguyen, T.T., Whalley, J., 1990. General equilibrium analysis of price controls: a computational approach. Int. Econ. Rev. 31, 667-684.

Ostrovsky, M., 2008. Stability in supply chain networks. Am. Econ. Rev. 98, 897-923.

Quinzii, M., 1984. Core and competitive equilibria with indivisibilities. Int. J. Game Theory 13, 41-60.

Roth, A.E., Sotomayor, M.A.O., 1990. Two-sided Matching, a Study in Game-theoretic Modeling and Analysis. Econom.

Soc. Monogr., vol. 18. Cambridge University Press, Cambridge.

Shapley, L.S., Scarf, H.E., 1974. On cores and indivisibility. J. Math. Econ. 1, 23-37.

Shapley, L.S., Shubik, M., 1971. The assignment game I: the core. Int. J. Game Theory 1, 111-130.

Sun, N., Yang, Z., 2006. Equilibria and indivisibilities: gross substitutes and complements. Econometrica 74, 1385-1402.

Talman, A.J.J., Yang, Z., 2008. A dynamic auction for differentiated items under price rigidities. Econ. Lett. 99, $278-281$.

Younès, Y., 1975. On the role of money in the process of exchange and the existence of a non-Walrasian equilibrium.

Rev. Econ. Stud. 42, 489-501. 